\title{
Molecular Dynamics simulation of
}

molecular crystals under anisotropic compression: bulk and directional effects in anthracene and paracetamol

Silvia Rizzato, ${ }^{\mathrm{a}}$ Angelo Gavezzotti, ${ }^{\mathrm{a}}$ Leonardo Lo Presti ${ }^{\mathrm{a}, *}$

${ }^{a}$ Department of Chemistry, Università degli Studi di Milano, Via Golgi 19, 20133 Milano (Italy)

*To whom correspondence should be addressed: leonardo.lopresti@unimi.it

\section{SUPPORTING INFORMATION}




\section{S1. Algorithm}

\section{S1.1. Detailed description of the pressure control algorithm}

The algorithm for applying arbitrary external stress to $N p T$ MD simulations was implemented in the Milano Chemistry Molecular Simulation (MiCMoS) package. The original algebra was developed by Parrinello \& Rahman ${ }^{1,2}$ and relies on Lagrangian dynamics applied to the $3 \times 3$ cell edge tensor, $\mathbf{H}$, that is, the array of three column vectors expressing cell edges Cartesian coordinates in the crystallophysical reference frame $\left\{\hat{\boldsymbol{e}}_{1,2,3}\right\}\left(\hat{\boldsymbol{e}}_{i} \cdot \hat{\boldsymbol{e}}_{j}=\delta_{i j} ;\left|\hat{\boldsymbol{e}}_{i, j, k}\right|=1\right)$. For the sake of simplicity, we associate the ordinary $\mathrm{X}, \mathrm{Y}$ and $\mathrm{Z}$ labels for the directions expressed by the $\hat{\boldsymbol{e}}_{1}, \hat{\boldsymbol{e}}_{2}$, and $\hat{\boldsymbol{e}}_{3}$ versors. We adopt a standard transform, pivoting on the crystallographic a cell axis, to define the laboratory Cartesian reference system, according to the orthogonalization procedure reported in Section A3 of the MiCMoS users' manual (https://sites.unimi.it/xtal_chem_group/images/MiCMoS_package/v1.1/MiCMoS v11 User Manual.pdf). In this frame, laboratory $\mathrm{X}, \mathrm{Y}$ and $\mathrm{Z}$ axes are exactly oriented as the cell edges $\mathbf{a}, \mathbf{b}$ and $\mathbf{c}$ if the crystallographic system has angles $\alpha=\beta=\gamma=90^{\circ}$. In monoclinic space groups, as anthracene and phase $I^{\circ}$ of paracetamol (see text), $\beta \neq 90^{\circ}$ and thus the $Z$ laboratory axis is not perfectly parallel to c. Different choices of the laboratory axes could be easily implemented in the source code, for example to apply an uniaxial stress field along specific crystallographic directions. The next release of the MiCMoS package will include a routine to allow the user to rotate the laboratory reference frame as he/she wish.

We start from the core equation of the PR algorithm for anisotropic pressure scaling

$$
\mathbf{F}=w \frac{d^{2} \mathbf{H}}{d t^{2}}=[\mathbf{P}-p \mathbf{I}] \boldsymbol{\sigma}
$$

Here, $\mathbf{F}$ is the force acting on the simulation box tensor $(\mathbf{H}), \mathbf{P}$ the pressure tensor as estimated from the virial field, $p$ the scalar (target) hydrostatic pressure, I the identity matrix and $\sigma$ the volume-scaled reciprocal cell matrix. $w$ is a coupling parameter with dimensions of a mass $(\mathrm{kg})$, which determines the inertial response of the lattice to the pressure unbalance. The external stress is added through a generalized $2^{\text {nd }}$-order symmetric tensor, $\mathbf{S}$, that is given in input by the user in units of GPa. The symmetry properties of the $\mathbf{S}$ matrix mirror the symmetry of the external stress field in the reference crystallophysical frame. For example, an external hydrostatic isotropic compression corresponds to $S_{11}=S_{22}=S_{33}$ and $S_{i j}=0 \forall i, j$ with magnitude equal to the trace of $\mathbf{S}(\operatorname{Tr}[\mathbf{S}])$, while any difference among the diagonal elements reflects into an anisotropic tensile stress along some laboratory direction(s) X, Y or Z. Nonzero off-diagonal elements $\left(S_{i j} \neq 0\right)$ correspond to applied shear stresses. According to the Cauchy notation, the index $i$ identifies the shear plane, taken as the one normal to the corresponding $i$-th axis, while $j$ specifies the laboratory axis along which the stress is applied. A symmetric tensor $\boldsymbol{\Sigma}$ is defined, which converts the applied stress, $\mathbf{S}$, into a surface energy density (force $/ \mathrm{m}$ or energy $/ \mathrm{m}^{2}$ ) by accounting for the shape of the unit cell.

$$
\boldsymbol{\Sigma}=\left[\mathbf{H}_{\mathbf{0}}^{-\mathbf{1}}(\mathbf{S}-p \mathbf{I}) \widetilde{\mathbf{H}}_{\mathbf{0}}^{-1}\right] \cdot V_{0}
$$

$\mathbf{H}_{0}$ here represent the starting (undistorted) simulation box tensor and the " -1 " and " $~ "$ symbols have the usual meaning of "inverse" and "transpose" matrix operations. Once the actual metric tensor $(\mathbf{G})$ is known, $\Sigma$ modifies the Lagrangian according to: 


$$
\mathcal{L}_{\text {stress }}=\mathcal{L}-\frac{1}{2} \operatorname{Tr}[\mathbf{\Sigma} \cdot \mathbf{G}]
$$

PR showed that this Lagrangian corresponds to the following equation of motion, which applies to the cell edge vectors and, indirectly, to all atom coordinates:

$$
\mathbf{F}=w \frac{d^{2} \mathbf{H}}{d t^{2}}=[\mathbf{P}-p \mathbf{I}] \boldsymbol{\sigma}-\mathbf{H} \cdot \mathbf{\Sigma}
$$

In practice, Eq. S4 is made compliant with standard leap-frog integrator used in MiCMoS. First, the velocity matrix $\mathbf{V}_{\mathbf{H}}$ associated to cell edge under a PR force $\mathbf{F}$ is estimated through:

$$
\mathbf{V}_{\mathbf{H}}=\frac{1}{2}\left(\frac{n \cdot \Delta t}{w}\right) \mathbf{F}
$$

In Eq. S5, $n$ is the number of MD steps between two anisotropic scaling procedures and $\Delta t$ the user-selected simulation time step. Finally, the Cartesian cell edge vectors $\mathbf{H}$ are updated:

$$
\mathbf{H}(t+\Delta t)=\mathbf{H}(t)+(n \cdot \Delta t) \mathbf{V}_{\mathbf{H}}
$$

Accordingly, all the molecules in the simulation box are rigidly translated to account for the cell edges displacement, $\Delta \mathbf{H}=\mathbf{H}(t+\Delta t)-\mathbf{H}(t)$.

\section{S1.2 Technical details}

\section{S1.2.1 Choice of the force field}

Both the force fields implemented in MiCMoS have their own strengths and weaknesses. The LJC parametrization employs atomic charges that are evaluated by fitting the MP2-derived molecu-lar electrostatic potential at the points of a grid defined according to Besler, Mertz \& Kollman. ${ }^{6}$ It has superior performances when medium or strong hydrogen bonds are structure-determining factors, such as in paracetamol. Indeed, calculations at $p=1$ bar showed that only the LJC functional gave the correct energy ranking of the two polymorphs. If the CLP force field is employed, the orthorhombic form II is erroneously predicted to be more stable than the monoclinic form I by $\sim 7 \mathrm{~kJ} / \mathrm{mol}$ at room $p$.

The CLP force field uses cheaper and less accurate Extended Hückel charges instead, in conjunction with a Coulomb-London-Pauli parametrization of dispersive-repulsive potentials. Its performances are generally good for systems dominated by van der Waals interactions, a fact that makes this parametrization a natural choice for modelling anthracene. If the LJC potentials are employed, agreement with experimental outcomes of cell parameters is significantly worse at high $p$ due to the unusual closeness of neighbouring molecules implies an overwighting of Coulomb terms with respect to the London ones.

\section{S1.2.2 Choice of the thermostat}

The Berendsen thermostat is known to introduce artefacts, as it suppresses energy fluctuations and causes an unphysical redistribution of kinetic energy into low frequency degrees of freedom at the expense of high frequency ones. ${ }^{3}$ In the present case, totally rigid (anthracene) or semi-rigid (paracetamol) solid state systems are considered. In paracetamol, only 3 flexible low frequency torsions per molecule are active (see Section S2.2 SI below), 
and their accessible phase space is even reduced due to closer and closer crystal packing at high pressure. Therefore, as already checked in previous works, ${ }^{4,5}$ this error can be safely ignored. Ultimately, the choice of Berendsen rescaling is justified by the satisfactory agreement with experiment for both the very different test cases here considered (see the main text).

\section{S1.3 Equivalence of different $p$ settings in the algorithm}

Figure S1 compares the effect of different $p$ coupling methods in the PR framework (equations 1 and 4 in the main text) for anthracene. Equilibration is very fast in both cases: results refer to the last frame of an explorative $200 \mathrm{ps}-$ long $N p T$ simulation at ambient pressure at $T=300 \mathrm{~K}$; data of the final crystallographic cells predicted after full $1 \mathrm{~ns}-$ long run can be found in Table S1 below. Pressure can be effectively fixed by both settings, which provide the same physical description of the system.

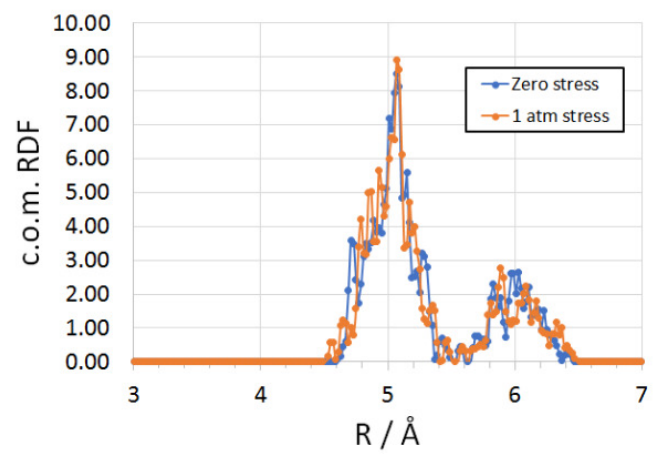

Figure S1. Centre of mass radial distribution function of anthracene at ambient conditions ( $p$ $=1 \mathrm{~atm}, \mathrm{~T}=300 \mathrm{~K}$ ), taken from the last frame of explorative $200 \mathrm{ps}-$ long MD NpT runs. The blue curve is obtained by exploiting Equation 1 with $p=1 \mathrm{~atm}$ and $w=3.0 \mathrm{~kg}$, the orange one with Equation 4 with $p=0, w=10 \mathrm{~kg}$ and and $S_{11}=S_{22}=S_{33}=1.0133 \cdot 10^{-4} \mathrm{GPa}, S_{12}=$ $\left.S_{13}=S_{23}=0.0 \mathrm{GPa}\right)$.

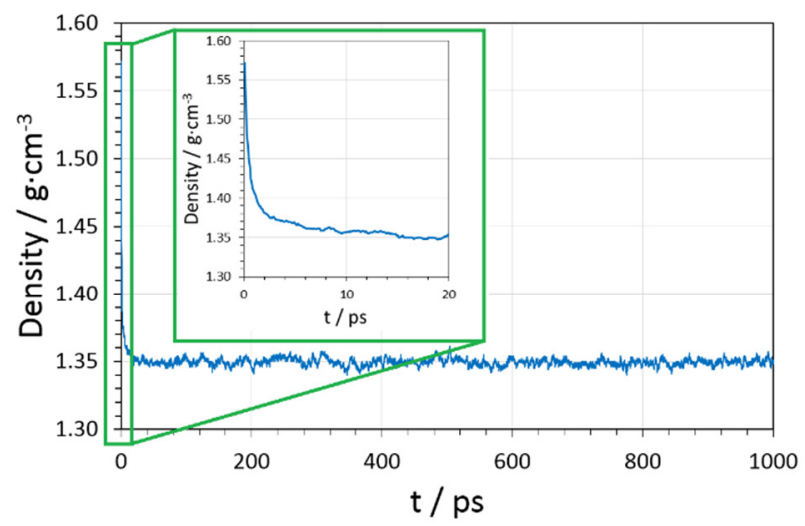

(a)

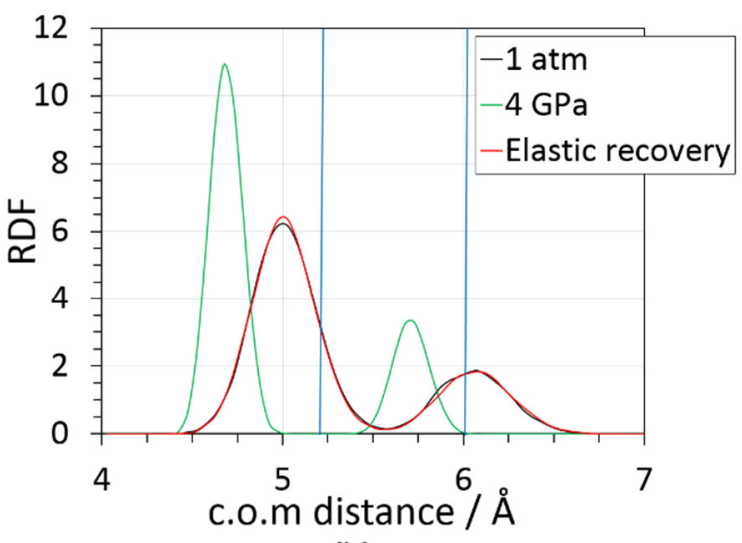

(b)

Figure S2. Elastic recovery of anthracene from $4 \mathrm{GPa}$ to ambient $p=1 \mathrm{~atm}$. (a) Lattice density as a function of the simulation time. In the first $20 \mathrm{ps}$, the system density decreases by more than $0.2 \mathrm{~g} \cdot \mathrm{cm}^{-3}$, becoming equal to that expected from the MD simulations in standard conditions. (b) Centre of mass (c.o.m.) radial distribution function (RDF) at $4 \mathrm{GPa}$ (green line) and after recovery from $4 \mathrm{GPa}$ to $1 \mathrm{~atm}$ (red line), compared with the structure equilibrated at $1 \mathrm{~atm}$ (black line). Vertical blue lines mark the c.o.m. for the experimental 
crystal structure at ambient conditions. All the distributions come from averages through the last 200 ps of the $1 \mathrm{~ns}-$-long trajectories.

We also verified whether MD calculations can simulate elastic recovery of the crystal structure of anthracene (Figure S2). To this end, we performed a regular $N p T$ MD run with the Parrinello-Rahman barostat $(w=3.0 \mathrm{~kg})$ and target $p=1 \mathrm{~atm}$, without external stress. The starting point was the equilibrated simulation box at $4 \mathrm{GPa}$. Upon relaxing the external stress, the unit cell very quickly recovers its original shape. The monoclinic angle $\beta$ closes by more than $3 \mathrm{deg}$ and the crystal density is reduced to $1.35 \mathrm{~g} \cdot \mathrm{cm}^{-3}$, which is the expected MD value for the ambient structure (see Table S1 below). This implies that deformation up to 4 $\mathrm{GPa}$ is fully elastic.

\section{S2. Input files}

See the MiCMoS user manual

(https://sites.unimi.it/xtal chem group/images/MiCMoS_package/v1.1/MiCMoS v11_User Manual.pdf) for full details on the meaning of all the quantities. CLP stands for all-atom Coulomb-London-Pauli Force Field ${ }^{4,5}$, LJC for Lennard-Jones-Coulomb. ${ }^{7}$

\section{S2.1 Topologies}

\section{S2.1.1 Anthracene}

\begin{tabular}{|c|c|c|c|c|c|c|c|}
\hline $\begin{array}{c}\text { \#ANTCEN } \\
24\end{array}$ & & $21 /$ & & topol & $\mathrm{AA}-\mathrm{CL}$ & & ce Field \\
\hline 1 & 0.0 & 609 & & .70372 & 3.65094 & 12 & -0.2922 \\
\hline 2 & 0.0 & 297 & & .42004 & 2.47909 & 12 & -0.3162 \\
\hline 3 & -0.0 & 440 & & .71390 & 1.21785 & 14 & 0.0475 \\
\hline 4 & -0.0 & 961 & & .41124 & -0.00916 & 12 & -0.3344 \\
\hline 5 & 0.0 & 310 & & .70977 & 1.22296 & 14 & 0.0498 \\
\hline 6 & -0.0 & 298 & & .40501 & 2.48445 & 12 & -0.3091 \\
\hline 7 & -0.0 & 579 & & .70747 & 3.65300 & 12 & -0.2911 \\
\hline 8 & -0.0 & 310 & & .70977 & -1.22296 & 14 & 0.0498 \\
\hline 9 & 0.0 & 961 & & .41124 & 0.00916 & 12 & -0.3344 \\
\hline 10 & 0.0 & 440 & & .71390 & -1.21785 & 14 & 0.0476 \\
\hline 11 & 0.0 & 298 & & .40501 & -2.48445 & 12 & -0.3091 \\
\hline 12 & -0.0 & 297 & & .42004 & -2.47909 & 12 & -0.3162 \\
\hline 13 & 0.0 & 579 & & .70747 & -3.65300 & 12 & -0.2911 \\
\hline 14 & -0.0 & 609 & & .70372 & -3.65094 & 12 & -0.2922 \\
\hline 15 & 0.0 & 787 & & .23257 & 4.59256 & 2 & 0.2872 \\
\hline 16 & 0.0 & 599 & & .49987 & 2.49979 & 2 & 0.2915 \\
\hline 17 & -0.0 & 863 & & .49121 & -0.01307 & 2 & 0.2901 \\
\hline 18 & -0.0 & 531 & & .48483 & 2.50303 & 2 & 0.2893 \\
\hline 19 & -0.0 & 721 & & .23989 & 4.59258 & 2 & 0.2877 \\
\hline 20 & 0.0 & 863 & & .49121 & 0.01307 & 2 & 0.2901 \\
\hline 21 & 0.0 & 531 & & .48483 & -2.50303 & 2 & 0.2893 \\
\hline 22 & -0.0 & 599 & & .49987 & -2.49979 & 2 & 0.2915 \\
\hline 23 & 0.0 & 721 & & .23989 & -4.59258 & 2 & 0.2877 \\
\hline 24 & -0.0 & 787 & & .23257 & -4.59256 & 2 & 0.2872 \\
\hline 0 & nsla & $-\mathrm{u}$ & & & & & \\
\hline 0 & ncor & & & & & & \\
\hline 0 & nsla & $-v$ & & & & & \\
\hline 172.6 & & $.0 \mathrm{v}$ & lu & $-\mathrm{u}$, volu & & & \\
\hline 26 & nstr & & & & & & \\
\hline 1 & 2 & 1.3 & & 5229.4 & $C-C$ & & \\
\hline 1 & 7 & 1.4 & & 4433.5 & $C-C$ & & \\
\hline 1 & 15 & 1.0 & & 3600.0 & $\mathrm{C}-\mathrm{H}$ & & \\
\hline 2 & 3 & 1.4 & & 3712.5 & $C-C$ & & \\
\hline 2 & 16 & 1.0 & & 3600.0 & $\mathrm{C}-\mathrm{H}$ & & \\
\hline 3 & 4 & 1.4 & & 4431.5 & $C-C$ & & \\
\hline 3 & 5 & 1.4 & & 4171.1 & $C-C$ & & \\
\hline 4 & 8 & 1.4 & & 4629.6 & $C-C$ & & \\
\hline 4 & 17 & 1.0 & & 3600.0 & $\mathrm{C}-\mathrm{H}$ & & \\
\hline 5 & 6 & 1.4 & & 3819.5 & $C-C$ & & \\
\hline
\end{tabular}




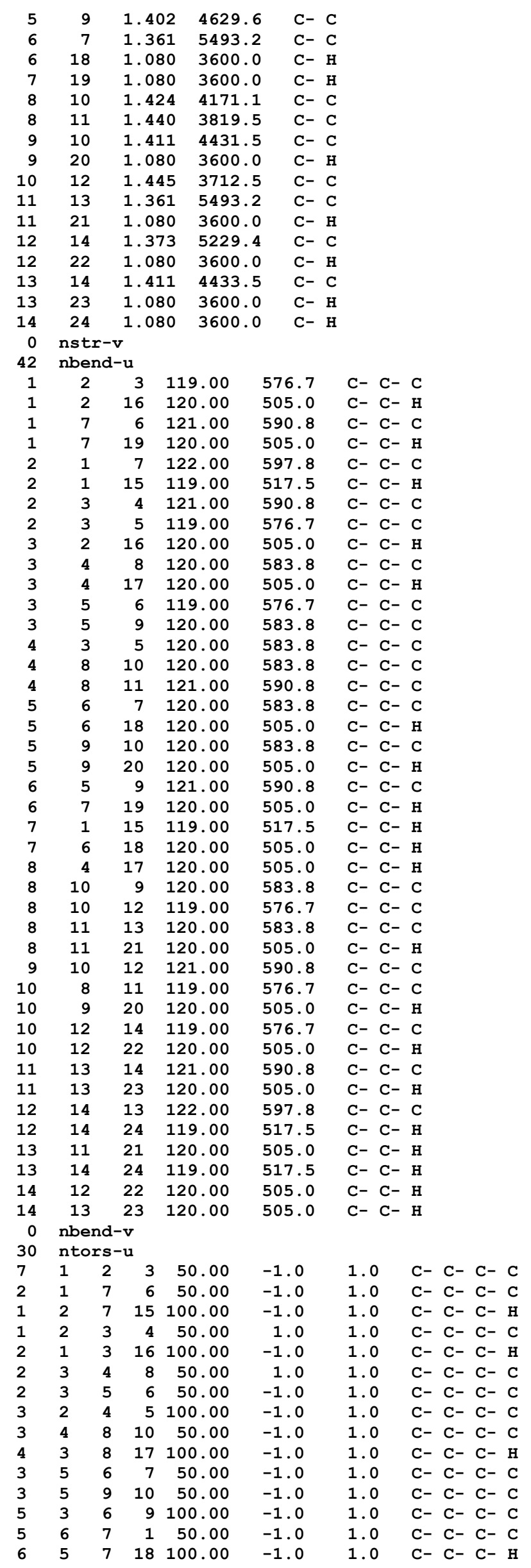




$\begin{array}{rrrrrrrl}7 & 1 & 6 & 19 & 100.00 & -1.0 & 1.0 & \text { C- C- C- H } \\ 4 & 8 & 10 & 9 & 50.00 & -1.0 & 1.0 & \text { C- C- C- C } \\ 4 & 8 & 11 & 13 & 50.00 & 1.0 & 1.0 & \text { C- C- C- C } \\ 8 & 4 & 10 & 11 & 100.00 & -1.0 & 1.0 & \text { C- C- C- C } \\ 5 & 9 & 10 & 8 & 50.00 & -1.0 & 1.0 & \text { C- C- C- C } \\ 9 & 5 & 10 & 20 & 100.00 & -1.0 & 1.0 & \text { C- C- C- H } \\ 8 & 10 & 12 & 14 & 50.00 & -1.0 & 1.0 & \text { C- C- C- C } \\ 10 & 8 & 9 & 12 & 100.00 & -1.0 & 1.0 & \text { C- C- C- C } \\ 8 & 11 & 13 & 14 & 50.00 & -1.0 & 1.0 & \text { C- C- C- C } \\ 11 & 8 & 13 & 21 & 100.00 & -1.0 & 1.0 & \text { C- C- C- H } \\ 10 & 12 & 14 & 13 & 50.00 & -1.0 & 1.0 & \text { C- C- C- C } \\ 12 & 10 & 14 & 22 & 100.00 & -1.0 & 1.0 & \text { C- C- C- H } \\ 11 & 13 & 14 & 12 & 50.00 & -1.0 & 1.0 & \text { C- C- C- C } \\ 13 & 11 & 14 & 23 & 100.00 & -1.0 & 1.0 & \text { C- C- C- H } \\ 14 & 12 & 13 & 24 & 100.00 & -1.0 & 1.0 & \text { C- C- C- H } \\ 0 & \text { ntors-v } & & & & \end{array}$

\section{S2.1.2 Paracetamol form $I^{\circ}$}

\begin{tabular}{|c|c|c|c|c|c|c|c|c|}
\hline $\begin{array}{c}\text { \# HXACAN } \\
20\end{array}$ & N27 & 21 & & topol & Logy LJC E & & $\mathrm{ce}$ & Field \\
\hline 1 & 0.0 & 299 & & .09834 & 2.55990 & & 12 & 0.366684 \\
\hline 2 & -0.1 & 847 & & .16454 & 1.68316 & & 12 & -0.213776 \\
\hline 3 & -0.2 & 079 & & .94928 & 0.30877 & & 12 & -0.249586 \\
\hline 4 & -0.1 & 060 & & .34426 & -0.20135 & & 12 & 0.338591 \\
\hline 5 & 0.0 & 561 & & .41329 & 0.68235 & & 12 & -0.243553 \\
\hline 6 & 0.1 & 273 & & .19766 & 2.05288 & & 12 & -0.300130 \\
\hline 7 & 0.0 & 679 & -0 & .14418 & -2.63331 & & 10 & 0.765967 \\
\hline 8 & -0.0 & 361 & & .52428 & -3.98140 & & 13 & -0.606869 \\
\hline 9 & 0.1 & 306 & & .36605 & 3.89860 & & 29 & -0.563428 \\
\hline 10 & -0.1 & 767 & & .66421 & -1.57989 & & 21 & -0.639100 \\
\hline 11 & 0.3 & 818 & & 35108 & -2.52309 & & 27 & -0.538898 \\
\hline 12 & -0.2 & 932 & & .16641 & 2.07371 & & 2 & 0.165855 \\
\hline 13 & -0.4 & 485 & & .78032 & -0.36125 & & 2 & 0.187639 \\
\hline 14 & 0.1 & 045 & & .41784 & 0.29503 & & 2 & 0.154914 \\
\hline 15 & 0.2 & 962 & & 03157 & 2.72540 & & 2 & 0.161152 \\
\hline 16 & -0.0 & 098 & & .59562 & -3.85012 & & 3 & 0.141486 \\
\hline 17 & 0.8 & 890 & & .26444 & -4.57714 & & 3 & 0.173856 \\
\hline 18 & -0.9 & 063 & & .19146 & -4.48234 & & 3 & 0.168811 \\
\hline 19 & -0.3 & 172 & & .63106 & -1.75698 & & 7 & 0.331540 \\
\hline 20 & -0.0 & 827 & & .45838 & 4.44109 & & 5 & 0.398848 \\
\hline 0 & nsla & & & & & & & \\
\hline 0 & ncor & & & & & & & \\
\hline 0 & nsla & $-v$ & & & & & & \\
\hline 140.7 & & $.0 \mathrm{v}$ & olu & $1-u$, volu & $1-v$ & & & \\
\hline 20 & nstr & & & & & & & \\
\hline 1 & 2 & 1.3 & & 4775. & $C-C$ & & & \\
\hline 1 & 6 & 1.3 & 96 & 4755.1 & $C-C$ & & & \\
\hline 1 & 9 & 1.3 & 68 & 4802.0 & $C-O$ & & & \\
\hline 2 & 3 & 1.3 & 93 & 4817.1 & $C-C$ & & & \\
\hline 2 & 12 & 1.0 & & 3600.0 & $\mathrm{C}-\mathrm{H}$ & & & \\
\hline 3 & 4 & 1.3 & 98 & 4722.0 & $C-C$ & & & \\
\hline 3 & 13 & 1.0 & & 3600.0 & $\mathrm{C}-\mathrm{H}$ & & & \\
\hline 4 & 5 & 1.3 & 98 & 4709.3 & $C-C$ & & & \\
\hline 4 & 10 & 1.4 & 16 & 4046.1 & $C-N$ & & & \\
\hline 5 & 6 & 1.3 & & 4901.6 & $C-C$ & & & \\
\hline 5 & 14 & 1.0 & & 3600.0 & $\mathrm{C}-\mathrm{H}$ & & & \\
\hline 6 & 15 & 1.0 & & 3600.0 & $\mathrm{C}-\mathrm{H}$ & & & \\
\hline 7 & 8 & 1.5 & & 2394.6 & $C-C$ & & & \\
\hline 7 & 10 & 1.3 & & 5693.0 & $C-N$ & & & \\
\hline 7 & 11 & 1.2 & & 7676.1 & $C-O$ & & & \\
\hline 8 & 16 & 1.0 & & 3600.0 & C- $\mathrm{H}$ & & & \\
\hline 8 & 17 & 1.0 & 80 & 3600.0 & $\mathrm{C}-\mathrm{H}$ & & & \\
\hline 8 & 18 & 1.0 & & 3600.0 & $\mathrm{C}-\mathrm{H}$ & & & \\
\hline 9 & 20 & 1.0 & & 4250.0 & $\mathrm{O}-\mathrm{H}$ & & & \\
\hline 10 & 19 & 1.0 & 00 & 5300.0 & $\mathrm{~N}-\mathrm{H}$ & & & \\
\hline 0 & nstr & & & & & & & \\
\hline 31 & nben & & & & & & & \\
\hline 1 & 2 & 3 & 12 & 1.00 & 590.8 & C- & C- & C \\
\hline 1 & 2 & 12 & 12 & 0.00 & 505.0 & $\mathrm{C}-$ & $\mathrm{C}-$ & $\mathrm{H}$ \\
\hline 1 & 6 & 5 & 12 & 0.00 & 583.8 & $\mathrm{C}-$ & $\mathrm{C}-$ & C \\
\hline
\end{tabular}




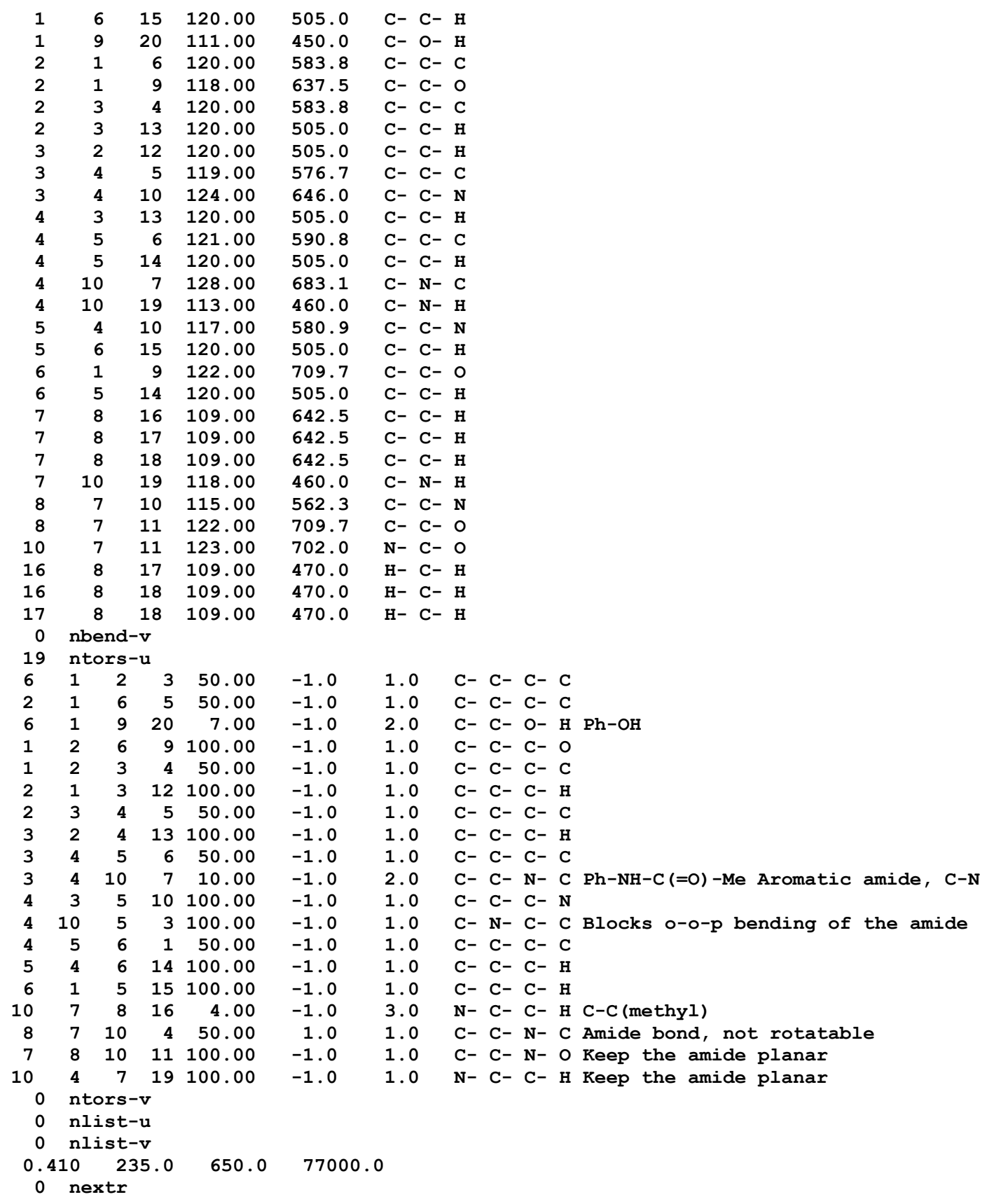

\section{S2.1.3. Paracetamol form II}

$\begin{array}{rrrrrr}\text { HXACAN33 'P b c a' topology LJC force field } \\ 20 & & & & & \\ 1 & -0.00351 & -0.10201 & -2.55834 & 12 & 0.347051 \\ 2 & -0.10564 & -1.16883 & -1.68340 & 12 & -0.201743 \\ 3 & -0.10780 & -0.96430 & -0.31852 & 12 & -0.273067 \\ 4 & -0.00985 & 0.33016 & 0.19186 & 12 & 0.385006 \\ 5 & 0.13760 & 1.38872 & -0.68771 & 12 & -0.274053 \\ 6 & 0.14617 & 1.17883 & -2.05031 & 12 & -0.265097 \\ 7 & 0.02232 & -0.13852 & 2.64837 & 10 & 0.789452 \\ 8 & -0.16671 & 0.55752 & 3.96337 & 13 & -0.582974 \\ 9 & -0.04175 & -0.33338 & -3.91056 & 29 & -0.557715 \\ 10 & -0.10955 & 0.64640 & 1.57385 & 21 & -0.672872 \\ 11 & 0.27650 & -1.34079 & 2.56109 & 27 & -0.553214 \\ 12 & -0.18455 & -2.17309 & -2.07292 & 2 & 0.162567 \\ 13 & -0.18529 & -1.80591 & 0.35395 & 2 & 0.197106 \\ 14 & 0.24726 & 2.39145 & -0.30187 & 2 & 0.157506 \\ 15 & 0.26976 & 2.01438 & -2.72336 & 2 & 0.152552 \\ 16 & 0.09019 & 1.60131 & 3.85843 & 3 & 0.139462\end{array}$




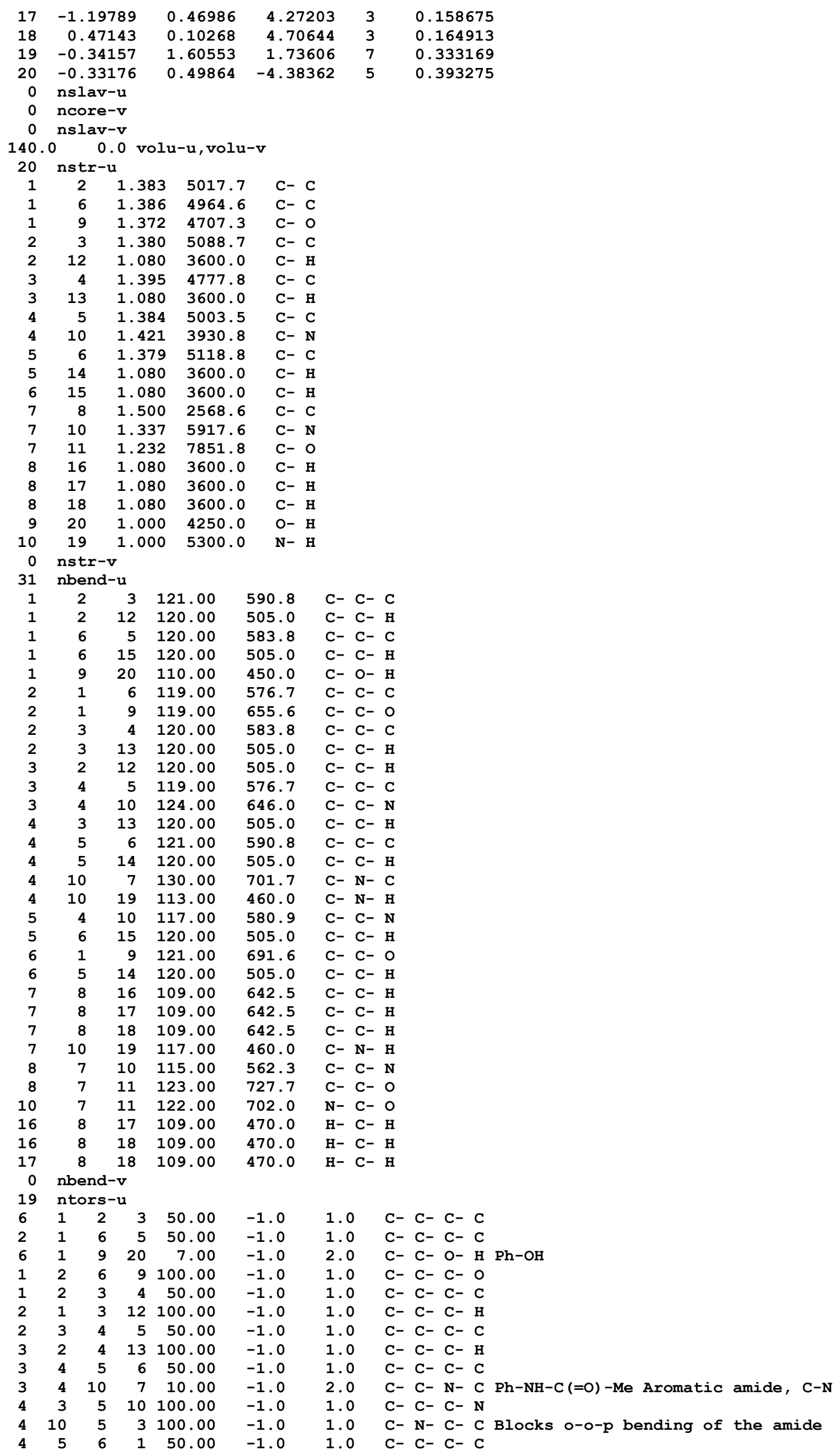




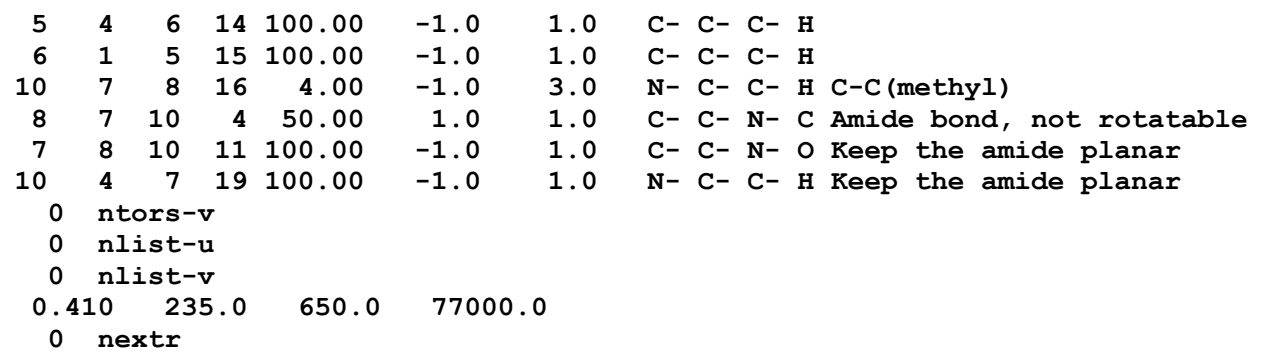

\section{S2.2 Input files, extension .mdi}

Note: the input file is independent on the crystal form and the same can be used for different polymorphs. Information on crystal forms is retrieved from topology (.top) and simulation box (.dat) files.

\section{S2.2.1 Anthracene, $p=1$ bar, no external stress field (1 ns, CLP, Parrinello-Rahman barostat for anistropic scaling of the unit cell)}

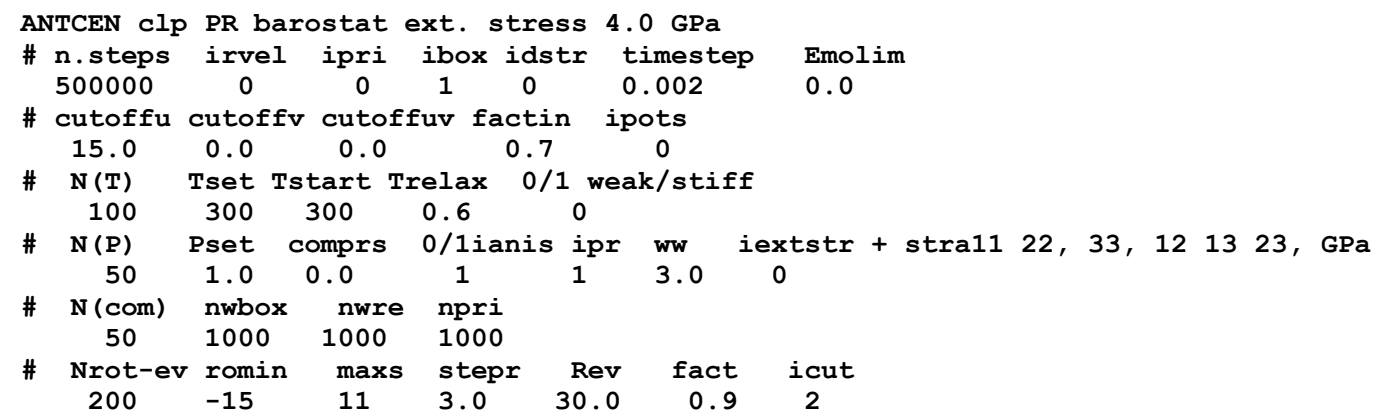

\section{S2.2.2 Anthracene, hydrostatic compression (4 GPa, 1 ns, CLP)}

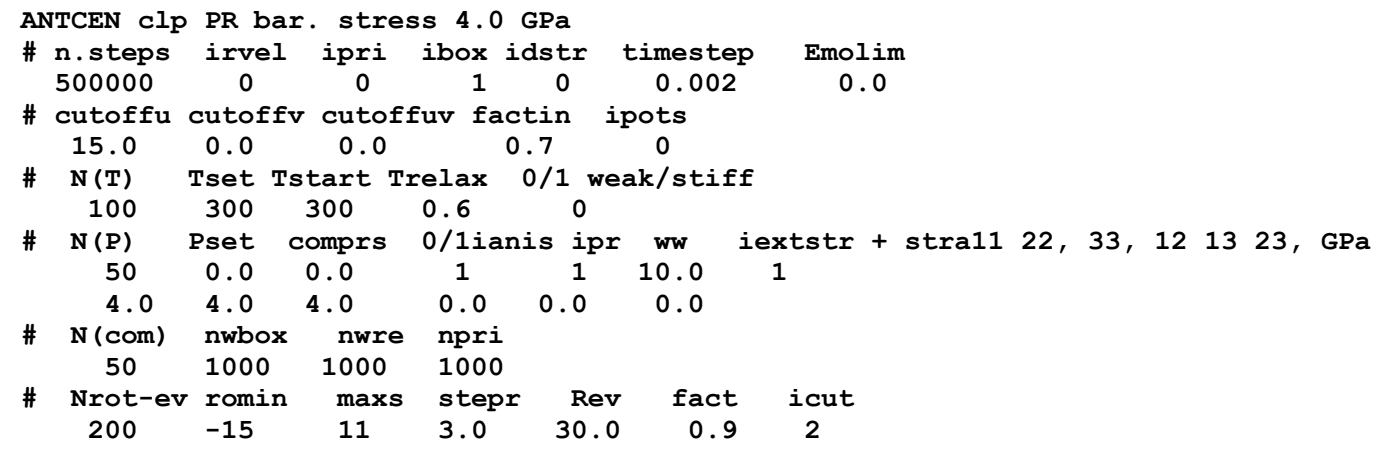

\section{S2.2.3 Anthracene, uniaxial compression of 0.5 GPa along Z (200 ps, CLP)}

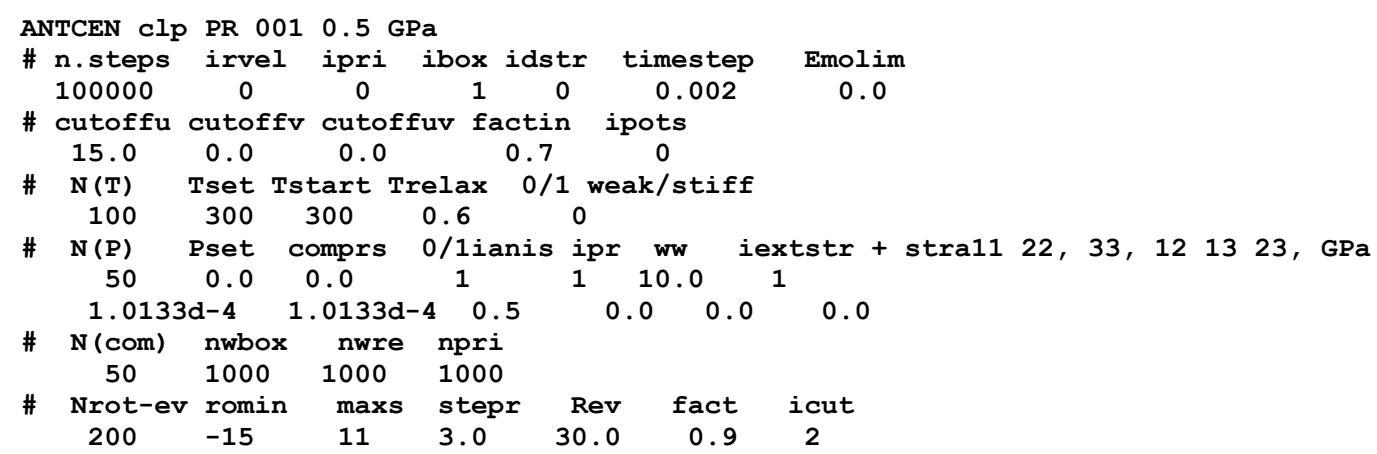


S2.2.4 Paracetamol, $p=1$ bar, no external stress field (1 ns, LJC, Parrinello-Rahman barostat for anistropic scaling of the unit cell)

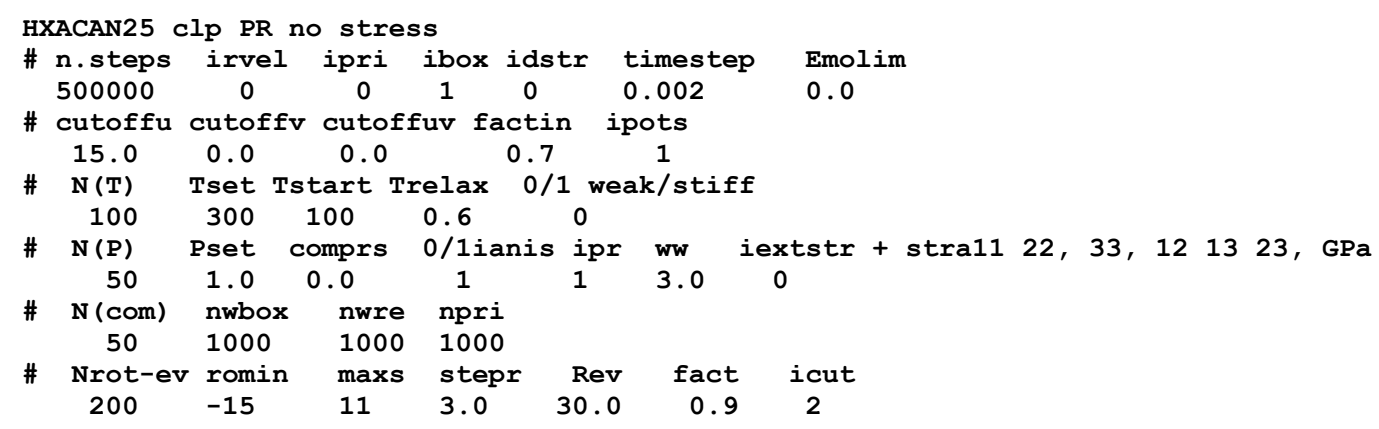

S2.2.5 Paracetamol, hydrostatic compression (4 GPa, 1 ns, LJC)

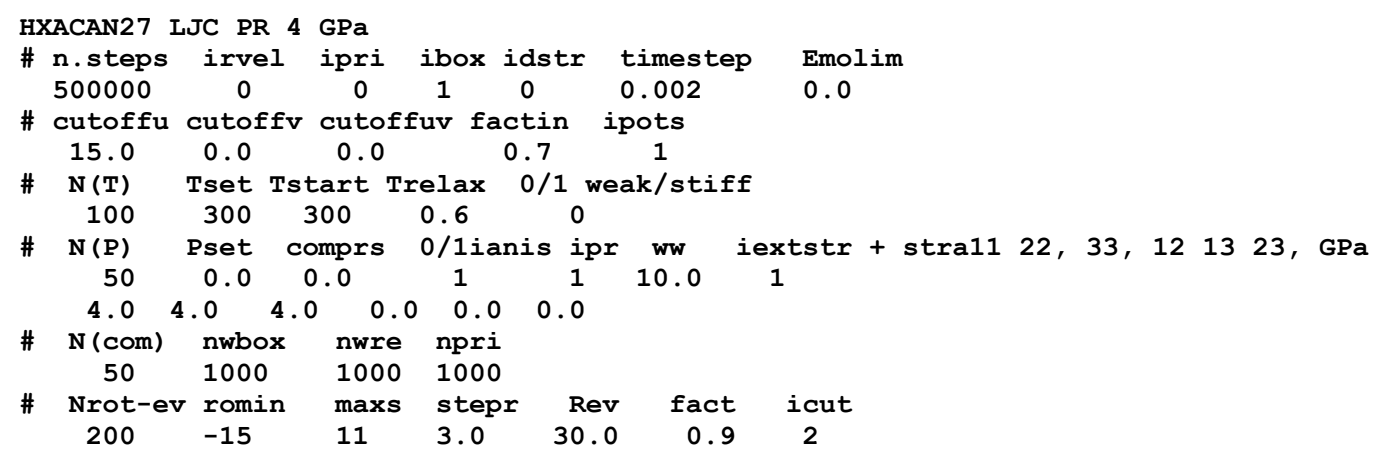

\section{S2.2.3 Paracetamol, uniaxial compression of 0.5 GPa along Z (200 ps, LJC)}

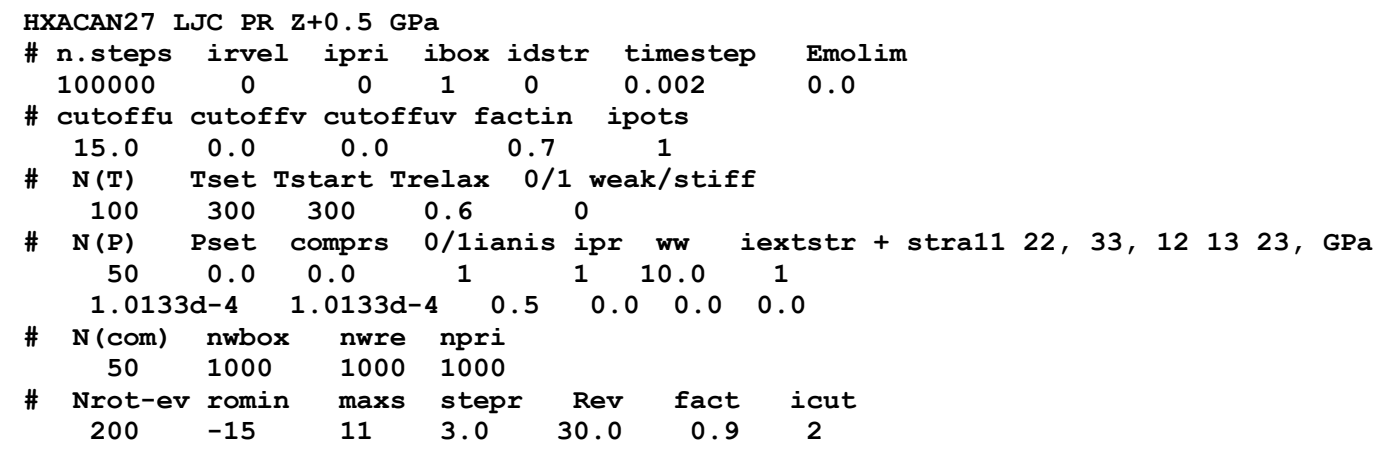




\section{S3. Results for anthracene}

\section{S3.1 Phase behaviour of polyacenes}

Anthracene (Scheme 1a, main text) is a prototypical molecular material for organic electrooptical devices. ${ }^{8}$ Polyacenes attract interest due to their noteworthy absorption and luminescence responses, coupled with large carrier mobility ${ }^{9}$. These properties all rely on extended aromatic $\pi$ systems. ${ }^{10}$ In the solid state, $\pi$ orbitals of neighbouring molecules overlap, resulting in strongly different absorption maxima and quantum yield with respect to solution. Any physically- or chemically-induced change in relative molecular distances and orientations mirrors a change of the lattice electronic states, giving thus unique opportunities to tune the corresponding macroscopic response. For example, it is known that the band gap of pentacene closes above $27 \mathrm{GPa}$, resulting in a strongly anisotropic metallic state at very high pressure ${ }^{11}$. As concerns anthracene, a detailed structural study up to $10 \mathrm{GPa}$ was carried out by Oezhelt, Resel \& Nakayama (ORN) ${ }^{12}$ with X-ray powder diffraction (XRPD) using $\mathrm{MeOH}: \mathrm{EtOH}$ 4:1 as pressure-transmitting medium.
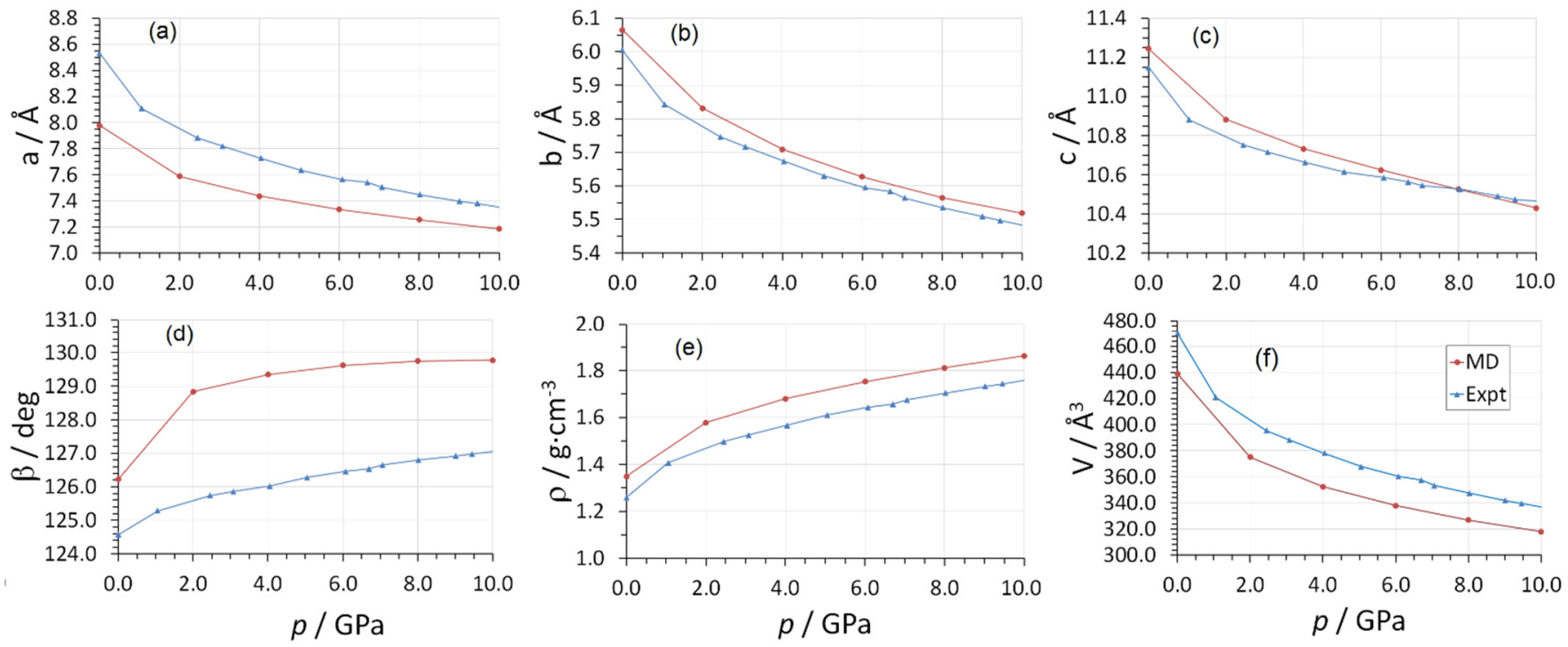

Figure S3. Predicted (red dots, this work) vs. experimental (blue triangles, PRB 2002, 66, 174104) cell edges ((a)-(c)), monoclinic angle (d), density (e) and volume (f) of anthracene up to $10 \mathrm{GPa}$. Predictions come from $1 \mathrm{~ns}-\operatorname{long} N p T$ MD simulations at $T=300 \mathrm{~K}$ with PR pressure scaling and an external isotropic stress matrix $\left(S_{\mathrm{ii}}=2,4,6,8,10 \mathrm{GPa}, S_{\mathrm{ij}}=0\right)$.

Broken lines serve only as a guide for the eye. 


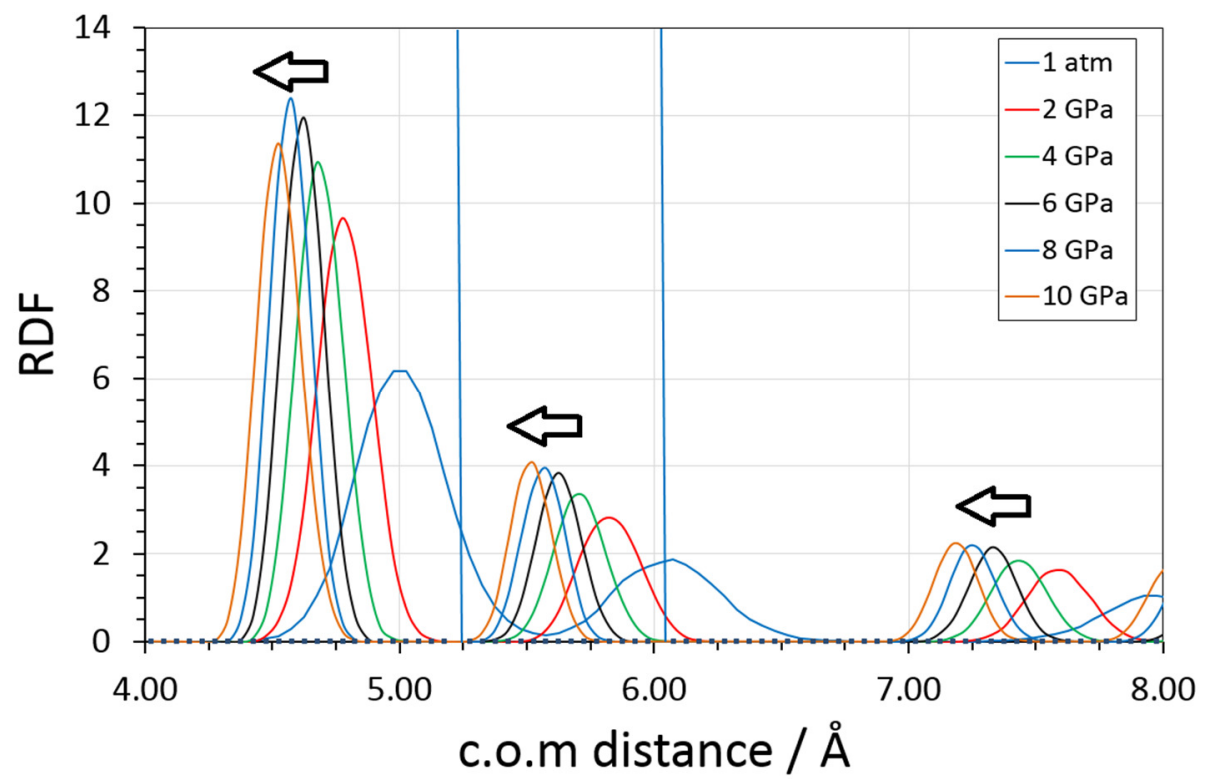

Figure S4. Centre of mass radial distribution function (c.o.m. RDF) for anthracene, averaged over the last 100 ps of the 1-ns long $N p T$ MD simulations, as a function of $p$. Black arrows show the shift of the RDF curve upon an increase of pressure.

Table S1. MD lattice parameters of anthracene $(\AA, \operatorname{deg})$, density $\left(\mathrm{g} / \mathrm{cm}^{3}\right)$ and cell volume $\left(\AA^{3}\right)$ as a function of the applied hydrostatic pressure. All the MD entries and the corresponding estimated standard deviations come from an average over the last $100 \mathrm{ps}$ of the 1 ns-long trajectory. See M. M. Julian, Foundations of Crystallography with Computer Applications, 2015, CRC Press, ISBN 9781466552913 for the corresponding experimental estimates.

\begin{tabular}{|c|c|c|c|c|c|c|c|c|c|}
\hline $\mathrm{p} / \mathrm{GPa}$ & $a / \AA$ & $b / \AA$ & $c / \AA$ & $\alpha / \operatorname{deg}$ & $\beta / \operatorname{deg}$ & $\gamma / \operatorname{deg}$ & $\rho / \mathrm{g} \cdot \mathrm{cm}^{-3}$ & $V / \AA^{3}$ & Notes \\
\hline $1.03 \mathrm{E}-04$ & 8.562 & 6.038 & 11.184 & 90 & 124.7 & 90 & 1.245 & 475.350 & Experimental, ${ }^{13}$ \\
\hline $1.03 \mathrm{E}-04$ & $7.977(9)$ & $6.061(6)$ & $11.242(8)$ & $90.05(5)$ & $126.23(9)$ & $89.92(6)$ & $1.350(3)$ & 438.420 & Elastic recovery ${ }^{a}$ \\
\hline $1.03 \mathrm{E}-04$ & $7.976(2)$ & $6.060(3)$ & $11.242(7)$ & $89.97(3)$ & $126.19(5)$ & $89.99(2)$ & $1.350(2)$ & 438.528 & 1 atm stress ${ }^{b}$ \\
\hline $1.03 \mathrm{E}-04$ & $7.978(6)$ & $6.065(6)$ & $11.245(1)$ & $90.05(7)$ & $126.23(6)$ & $89.95(5)$ & $1.349(2)$ & 438.927 & Zero stress ${ }^{c}$ \\
\hline 2 & $7.588(2)$ & $5.832(2)$ & $10.883(3)$ & $89.97(3)$ & $128.84(4)$ & $89.85(3)$ & $1.578(1)$ & 375.143 & $\mathrm{~S}_{\mathrm{ii}}=2, \mathrm{~S}_{\mathrm{ij}}=0.0, w=10 \mathrm{~kg}$ \\
\hline 4 & $7.437(4)$ & $5.709(3)$ & $10.733(3)$ & $89.95(5)$ & $129.35(3)$ & $89.87(5)$ & $1.680(1)$ & 352.379 & $\mathrm{~S}_{\mathrm{ii}}=4, \mathrm{~S}_{\mathrm{ij}}=0.0, w=10 \mathrm{~kg}$ \\
\hline 6 & $7.335(4)$ & $5.627(2)$ & $10.624(3)$ & $89.91(5)$ & $129.62(2)$ & $89.88(5)$ & $1.753(1)$ & 337.761 & $\mathrm{~S}_{\mathrm{ii}}=6, \mathrm{~S}_{\mathrm{ij}}=0.0, w=10 \mathrm{~kg}$ \\
\hline 8 & $7.256(3)$ & $5.565(2)$ & $10.526(5)$ & $89.87(5)$ & $129.75(5)$ & $89.86(3)$ & $1.811(1)$ & 326.781 & $\mathrm{~S}_{\mathrm{ii}}=8, \mathrm{~S}_{\mathrm{ij}}=0.0, w=10 \mathrm{~kg}$ \\
\hline 10 & $7.185(3)$ & $5.519(3)$ & $10.430(5)$ & $89.96(9)$ & $129.78(5)$ & $89.83(6)$ & $1.862(1)$ & 317.840 & $\mathrm{~S}_{\mathrm{ii}}=10, \mathrm{~S}_{\mathrm{ij}}=0.0, w=10 \mathrm{~kg}$ \\
\hline
\end{tabular}

${ }^{a}$ Anisotropic pressure scaling with reference $p=1 \mathrm{~atm}$ (PR algorithm, Eq. 1 in the main text), with no external stress applied. The starting point was the structure equilibrated at $4 \mathrm{GPa}($ Model \#6).

${ }^{b}$ Anisotropic pressure scaling with reference $p=0 \mathrm{~atm}$ and an external $1 \mathrm{~atm}$ stress applied (PR algorithm, Eq. 7 in the main text).

${ }^{c}$ Anisotropic pressure scaling with reference $p=1$ atm (PR algorithm, Eq. 1 in the main text), starting from the experimental structure. 


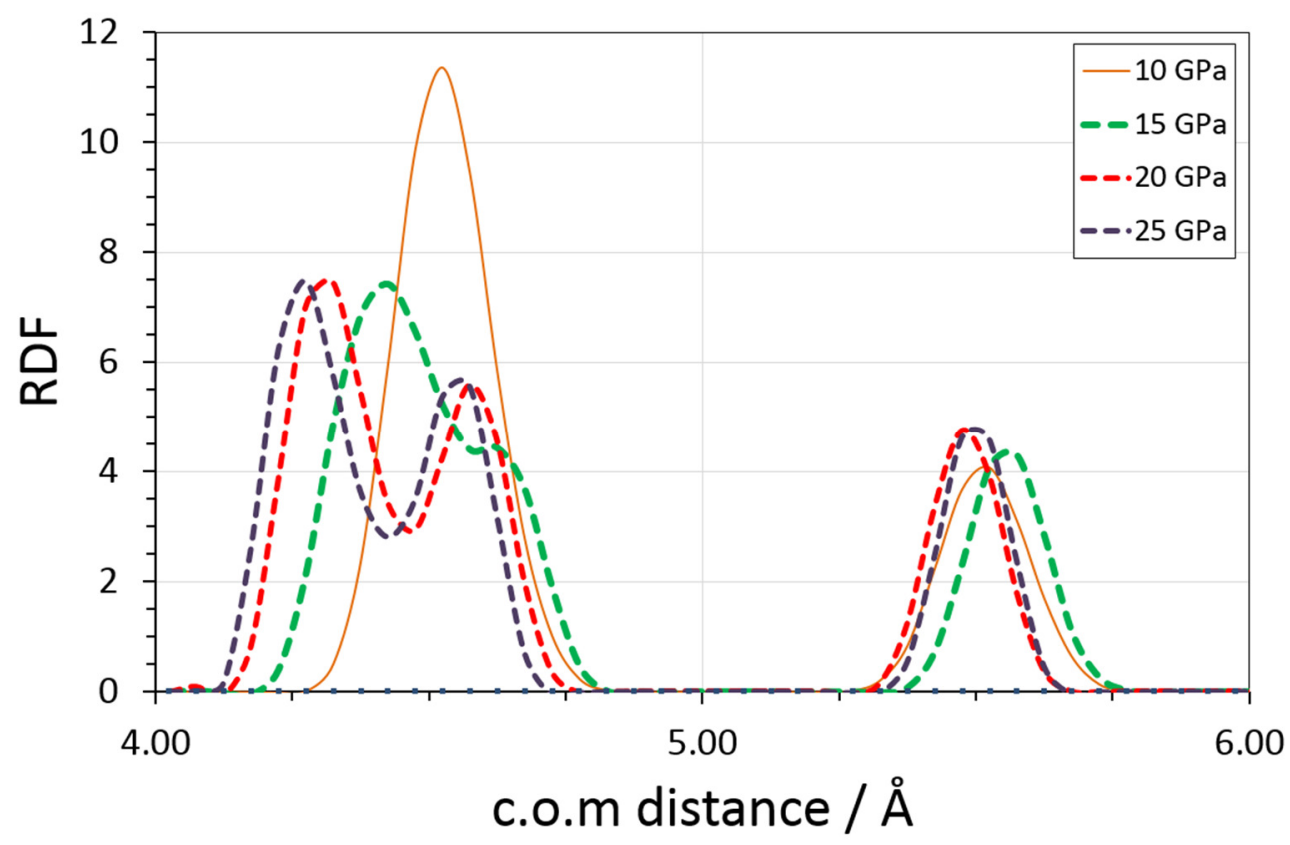

Figure S5. Same as Figure S3, for very high (>10 GPa) hydrostatic compression (dashed curves). A progressive reduction of symmetry in the first coordination shell is detectable, with average c.o.m. distances splitting in two groups. This difference, however, does not imply a structural phase transition (Figure S6).
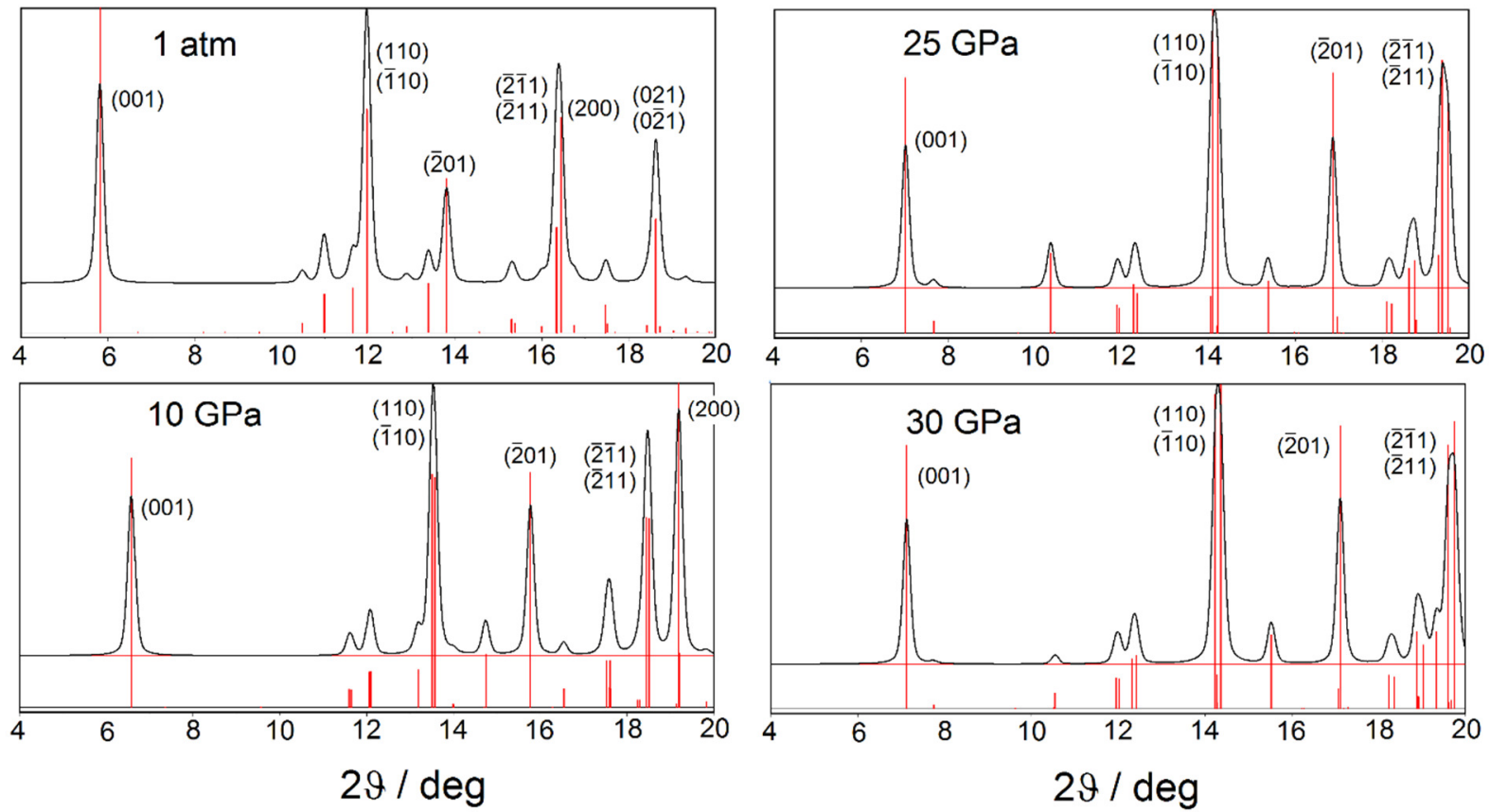

Figure S6. Simulated X-ray powder patterns of anthracene under isotropic hydrostatic stress, in the $1 \mathrm{~atm}-30 \mathrm{GPa}$ range of pressure. $(\mathrm{hkl})$ indices of intense Bragg reflections are highlighted. Intensity is on arbitrary units. The diffraction profiles ${ }^{12}$ refer to $\lambda=0.9204 \AA$ with pseudo-Voigt functions $(\eta=0.5)$ and full-width at half maximum of $0.2 \mathrm{deg}$. Despite the change in the first coordination shell of anthracene above $p=15 \mathrm{GPa}$ (Figures S5 and S7), no phase transitions occur, and the average structure stays $P 2_{1} / a$ throughout the whole 1 atm $-30 \mathrm{GPa}$ range of $p$. 


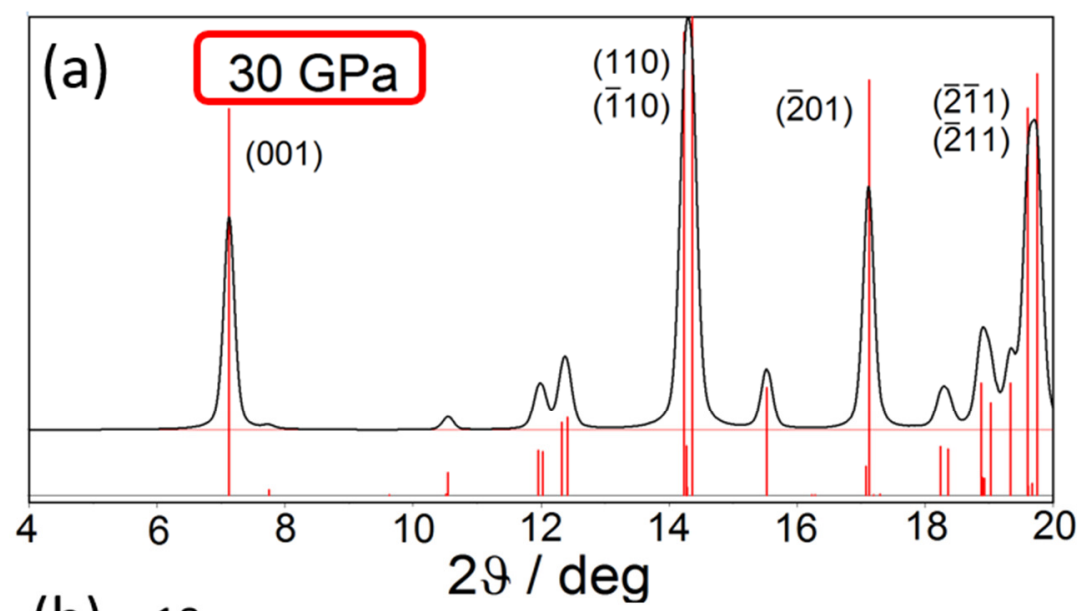

(c)

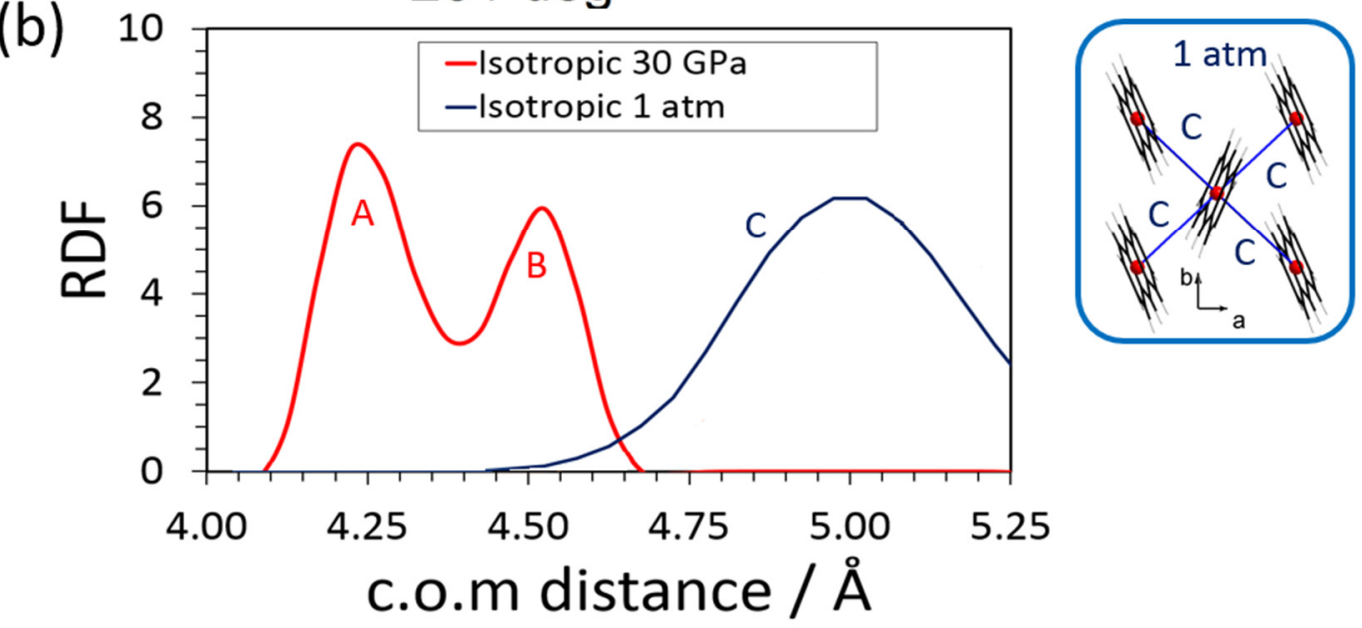

Figure S7. (a) Predicted X-ray powder diffraction output from the structure averaged over the last 200 ps of the $1 \mathrm{~ns}-$ long trajectory at $p=30 \mathrm{GPa}$, assuming $\lambda=0.9204 \AA^{12}$ with pseudo-Voigt functions $(\eta=0.5)$ and full-width at half maximum of $0.2 \mathrm{deg}$.

Crystallographic indices $(h k l)$ of Bragg-reflected relevant planes are highlighted. (b) C.o.m. RDF of the structure equilibrated at $30 \mathrm{GPa}$ (red curve), compared with the ambient (1 atm, blue curve) one. (c) Packing arrangement of the first coordination shell of anthracene, explaining the differences in centre of mass distances highlighted by RDF in (b). 

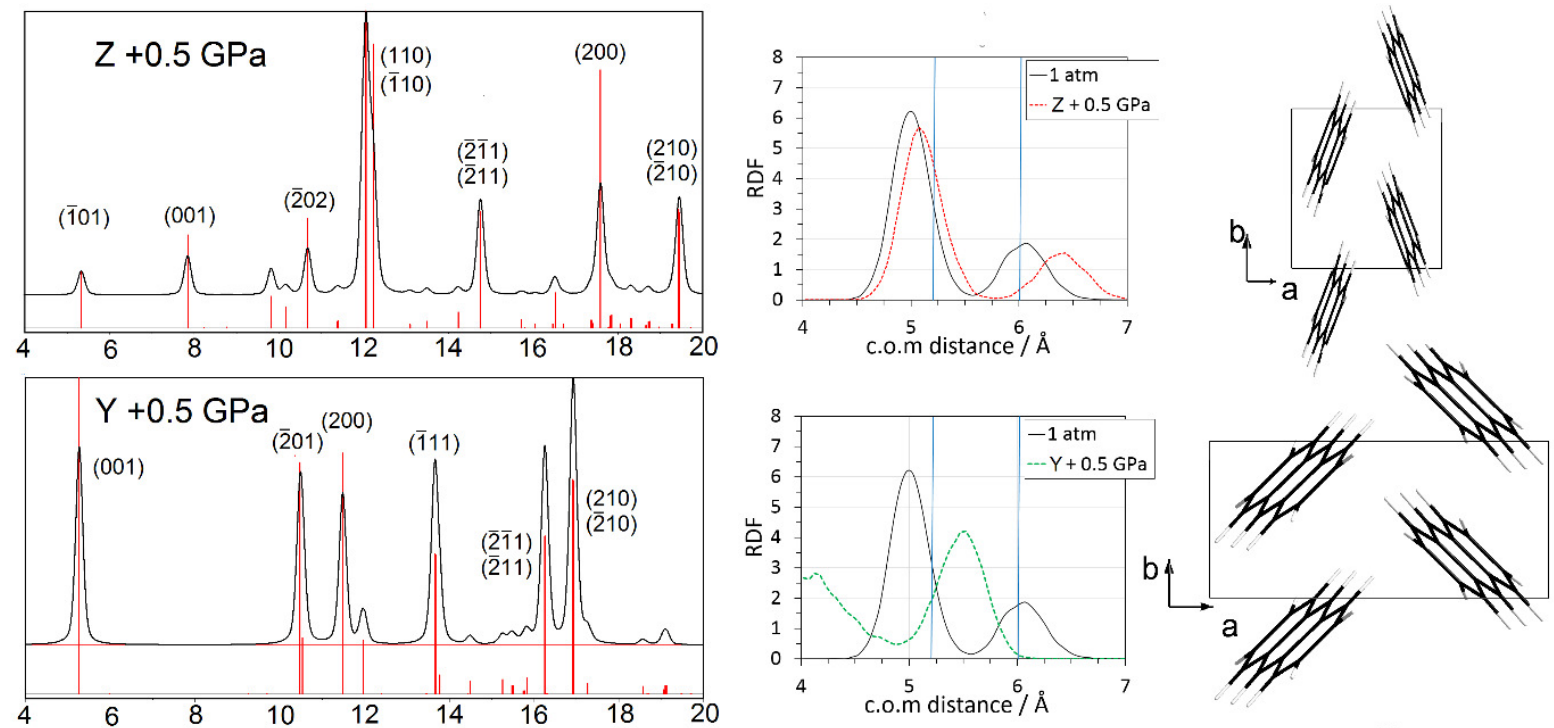

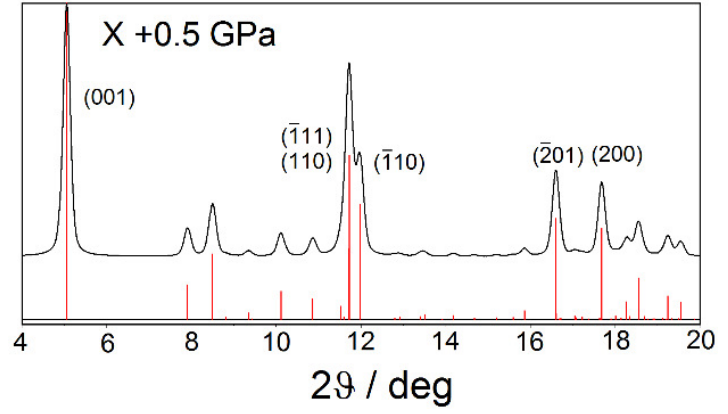

(a)

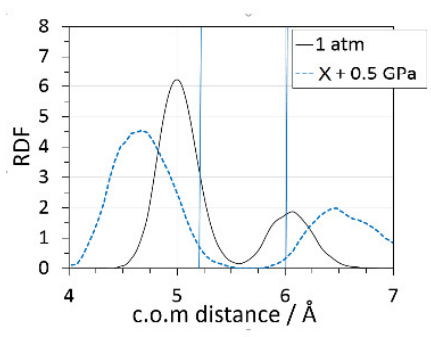

(b)

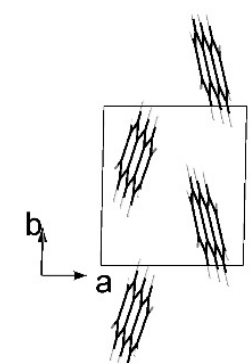

(c)

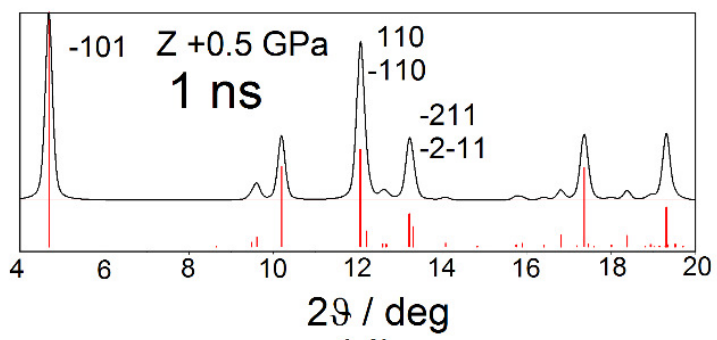

(d)

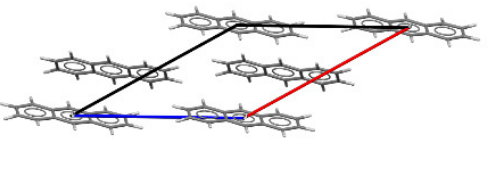

(e)

Figure S8. (a) Simulated X-ray powder patterns of anthracene under tensile compression of $0.5 \mathrm{GPa}$, along the Z, Y and X laboratory axes. Significant structural rearrangements occur in each case, a testified by shifts of and intensity changes with respect to the reference structure (Figure S6). A clear evidence of symmetry breaking is the ( 101$)$ reflection, forbidden in $P 2_{1} / a$, which appears when the stress is applied along Z. (b) Centre-of-mass Radial Distribution Function of the corresponding anisotropically stressed structure (dotted lines), as compared with the $p=1$ atm (black curve) and the ideal crystallographic (vertical bars) ones. (c) Crystal packing along the $c$ axis. Wider RDF peaks correspond to noisier XRPD signals, implying some crystallinity loss. The Z-compressed structure is the most similar to the isotropically compressed ones (Figure S6), despite the loss of the $a$ glide plane (see text). (d) Same as (a), after extending the simulation to $1 \mathrm{~ns}$. The ( $\overline{101})$ reflection has now become much larger, reflecting a higher degree of deformation of the polar $P 2_{1}$ structure at high-p. (e) Unit cell of anthracene averaged through the last 100 ps of a 1 ns-long trajectory under uniaxial compression of $0.5 \mathrm{GPa}$ along $\mathrm{Z}$. View along the $b$ axis as in Figure 2 (main text). 
S3.1 Z+0.5 model (Figure S8a, average structure in P1)

$\begin{array}{lllllrrr}\text { TITL MD averaged structure } & Z+0.5 & \text { (no symmetry) } & 200 & \text { ps-long trajectory } \\ \text { CELL } & 0.71073 & 10.0196 & 6.4065 & 11.1937 & 89.9715 & 143.0606 & 89.9711 \\ \text { ZERR } & 1 & 0.0239 & 0.0058 & 0.0071 & 0.0066 & 0.0653 & 0.0069\end{array}$

\begin{tabular}{|c|c|c|}
\hline \multicolumn{2}{|c|}{ ZERF } & 0.0239 \\
\hline
\end{tabular}

0.76759

0.89599

0.82340

0.95413

0.61661

0.48797

0.88025

0.54287

0.67439

1.01008

0.60166

0.93352

0.73059

0.82547

1.04660

1. 10852

0.47446

0.38931

1. 16652

0.45042

1.02443

0.67358

0.29125

0.13917

$\begin{array}{ll}\text { H2 } & 2 \\ \text { MOLE } & 2\end{array}$

C1 1

C2 1

0.66714

0.63573

0.69008

0.66755

0.76844

0.79227

0.74796

0.72407

0.82631

0.80483

0.69949

0.86004

0.74405

0.82582

0.63410

0.57534

0.60693

0.84418

0.78628

0.88598

0.65132

0.92173

0.70625

0.85694

424

HKLF 4

END

0.27034

0.39895

0.32515

0.45555

0.11808

$-0.01063$

0.06654

0.38060

0.04336

0.17416

0.50873

0.10054

0.43147

0.22804

0.32759

0.55064

0.60920

$-0.16709$

$-0.02372$

$-0.11065$

0.66570

$-0.05026$

0.52180

0.16960
0.56489

0.33113

0.71098

0.63905

0.48150

0.39722

0.41156

0.49660

0.63741

0.24361

0.25709

0.17165

0.15781

0.01532

0.01596

$-0.05653$

0.82015

0.69549

0.44937

0.45079

0.68458

0.20326

0.20333

$-0.03969$

$-0.03233$

$-0.16518$

$-0.12685$

$-0.05379$

0.10228

0.18602

0.17197

0.08559

$-0.05583$

0.33947

0.32611

0.41063

0.42505

0.56742

0.56676

0.63861

$-0.23673$

$-0.10911$

0.13278

0.13257

$-0.10470$

0.37898

0.38051

0.62362

0.61568

0.74676

11.00000

11.00000

11.00000

11.00000

11.00000

11.00000

11.00000

11.00000

11.00000

11.00000

11.00000

11.00000

11.00000

11.00000

11.00000

11.00000

11.00000

11.00000

11.00000

11.00000

11.00000

11.00000

11.00000

11.00000

0.05

0.05

0.05

0.05

0.05

0.05

0.05

0.05

0.05

0.05

0.05

0.05

0.05

0.05

0.05

0.05

0.05

0.05

0.05

0.05

0.05

0.05

0.05

$11.00000 \quad 0.05$

$11.00000 \quad 0.05$

$11.00000 \quad 0.05$

$11.00000 \quad 0.05$

$11.00000 \quad 0.05$

$11.00000 \quad 0.05$

$11.00000 \quad 0.05$

$11.00000 \quad 0.05$

$11.00000 \quad 0.05$

$11.00000 \quad 0.05$

$11.00000 \quad 0.05$

$11.00000 \quad 0.05$

$11.00000 \quad 0.05$

$11.00000 \quad 0.05$

$11.00000 \quad 0.05$

$11.00000 \quad 0.05$

$11.00000 \quad 0.05$

$11.00000 \quad 0.05$

$11.00000 \quad 0.05$

$11.00000 \quad 0.05$

$11.00000 \quad 0.05$

$11.00000 \quad 0.05$

$\begin{array}{ll}11.00000 & 0.05\end{array}$

$11.00000 \quad 0.05$ 


\section{PLATON ADDSYM output:}

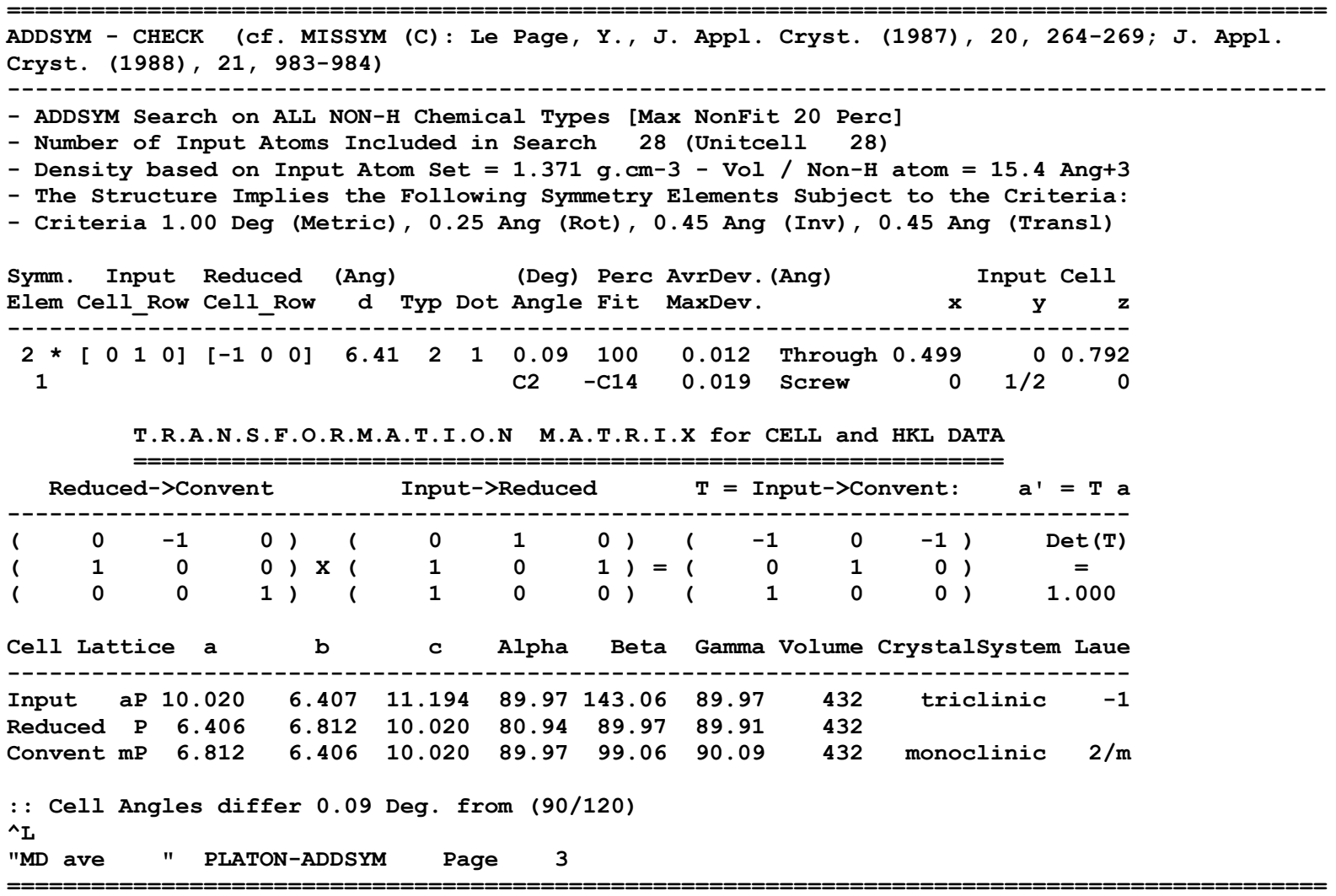

Conventional, New or Pseudo Symmetry

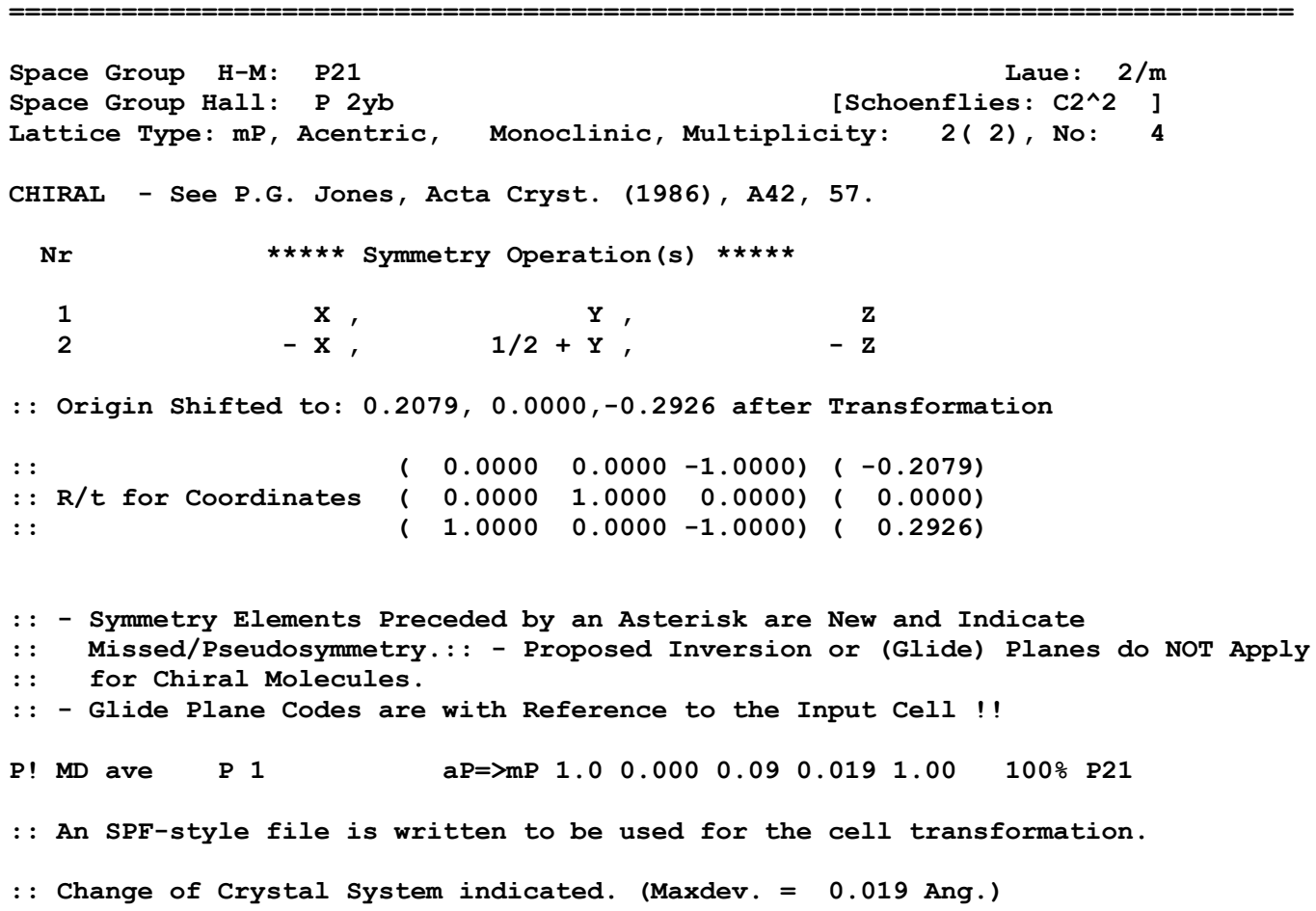




\section{S4.1 Phase behaviour of paracetamol}

Also known as acetaminophen, paracetamol is a major painkiller with a good safety profile, despite its not entirely known mechanism of action. ${ }^{14}$ At least six different crystalline forms were reported to date, four of which were fully characterized by X-ray crystallography. The monoclinic $\mathrm{I}^{\mathrm{O}}\left(\mathrm{P} 2{ }_{1} / \mathrm{n}, \mathrm{m} . \mathrm{p} .171{ }^{\circ} \mathrm{C}\right)$ and the metastable orthorhombic II (Pbca, m.p. $\left.157{ }^{\circ} \mathrm{C}\right)$ polymorphs ${ }^{1}$ are the most deeply studied ones ${ }^{15-17}$. They are enantiotropic, with partial $\mathrm{II} \rightarrow \mathrm{I}^{\mathrm{O}}$ conversion above $\mathrm{T}=122{ }^{\circ} \mathrm{C} .{ }^{18}$ Recent high-quality XRPD experiments found that the elusive and highly metastable form III (m.p. $143^{\circ} \mathrm{C}$ ), ${ }^{19}$ first discovered in $80 \mathrm{~s},{ }^{20}$ crystallizes in $P c a 2_{1}{ }^{21,22}$ or $P n a 2_{1}{ }^{23}$, and undergoes a reversible phase transition to a monoclinic $P c$ form $(\mathrm{III}-m)$ below $\mathrm{T}=220 \mathrm{~K}^{22}$. Recently, joint Raman and powder X-ray experiments ${ }^{24}$ reported the occurrence of two further high- $p$ forms, IV and V, whose crystal structures are still unknown. Amorphous paracetamol can be also obtained by rapidly cooling the melt ${ }^{25}$.

This rich crystallization landscape is due to the highly functionalized nature of the compound (see Scheme 1 in the main text). The presence of hydrogen bond donors $(\mathrm{NH}, \mathrm{OH})$ and acceptors $(\mathrm{C}=\mathrm{O}, \mathrm{OH})$ provides the opportunity of playing with competing molecular recognition modes, i.e. by tuning the crystallization conditions with suitable additives ${ }^{26}$. Two high $-p$ X-ray studies of paracetamol I ${ }^{\mathrm{o}}$ (single crystal) and II (powder) were carried out in 2000 by Boldyreva and co-workers up to $4 \mathrm{GPa}^{27,28}$, with the aim of clarifying the role of different HB patterns in determining anisotropic compressibility of the corresponding lattice parameters. We will also focus on such two phases, as no high-p crystallographic data are available for the other polymorphs.

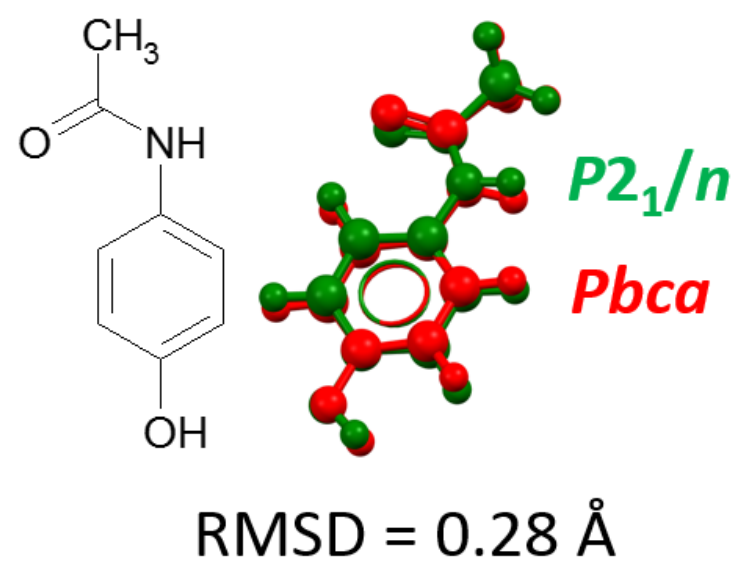

Figure S9. Similarity of the molecular conformations in polymorphs $\mathrm{I}^{\circ}$ and II, as estimated from single crystal $\mathrm{X}$-ray diffraction. The root mean square deviation among non- $\mathrm{H}$ atoms is also shown.

\footnotetext{
${ }^{1}$ The Kofler notation ${ }^{31}$ is employed throughout to label different polymorphs by Roman numerals with melting points in decreasing order. The ${ }^{\mathrm{c}}$, symbol marks the most stable form
} 


\section{S4.2 Results for paracetamol}

Table S2. MD-predicted lattice parameters (LJC potential) for paracetamol (form I ${ }^{\circ}, P 2_{1} / n$ ) as a function of the applied hydrostatic pressure. Each entry comes from the average of the last $200 \mathrm{ps}$ of $1 \mathrm{~ns}-$ long trajectories. All the calculations were performed under $\mathrm{NpT}$ control with the Parrinello-Rahman algorithm at $T=300 \mathrm{~K}$.

\begin{tabular}{l|llllllll}
\hline$p / \mathrm{GPa}$ & $a / \AA$ & $b / \AA$ & $c / \AA$ & $\alpha / \mathrm{deg}$ & $\beta / \mathrm{deg}$ & $\gamma / \mathrm{deg}$ & $\rho / \mathrm{g} \cdot \mathrm{cm}^{-3}$ & $V / \AA^{3}$ \\
\hline $1.03 \mathrm{E}-04^{a}$ & $7.0915(3)$ & $9.2149(4)$ & $11.6015(5)$ & 90. & $97.865(1)$ & 90. & 1.3370 & 750.997 \\
$1.03 \mathrm{E}-04^{b}$ & $6.891(4)$ & $9.924(6)$ & $11.242(13)$ & $90.184(66)$ & $95.849(79)$ & $89.854(28)$ & $1.313(2)$ & 764.790 \\
1 & $6.795(2)$ & $9.340(2)$ & $10.963(4)$ & $89.814(37)$ & $96.327(29)$ & $89.791(15)$ & $1.452(1)$ & 691.523 \\
2 & $6.713(2)$ & $9.116(1)$ & $10.760(4)$ & $89.808(41)$ & $96.253(42)$ & $89.774(23)$ & $1.534(1)$ & 654.539 \\
3 & $6.633(2)$ & $8.975(2)$ & $10.621(4)$ & $89.769(35)$ & $96.095(38)$ & $89.750(25)$ & $1.597(1)$ & 628.694 \\
4 & $6.553(2)$ & $8.876(2)$ & $10.517(4)$ & $89.736(35)$ & $95.837(52)$ & $89.769(27)$ & $1.650(1)$ & 608.531 \\
\hline
\end{tabular}

${ }^{a}$ Experimental. $^{29}$

${ }^{b}$ No external stress applied.

Table S3. MD-predicted lattice parameters (LJC potential) for paracetamol (form II, $\mathrm{Pbca}$ ) as a function of the applied hydrostatic pressure. Each entry comes from the average of the last $200 \mathrm{ps}$ of $1 \mathrm{~ns}-$ long trajectories. All the calculations were performed under $\mathrm{NpT}$ control with the Parrinello-Rahman algorithm at $T=300 \mathrm{~K}$.

\begin{tabular}{l|llllllll}
\hline$p / \mathrm{GPa}$ & $a / \AA$ & $b / \AA$ & $c / \AA$ & $\alpha / \operatorname{deg}$ & $\beta / \operatorname{deg}$ & $\gamma / \mathrm{deg}$ & $\rho / \mathrm{g} \cdot \mathrm{cm}^{-3}$ & $V / \AA^{3}$ \\
\hline $1.03 \mathrm{E}-04^{a}$ & $11.8237(4)$ & $7.3971(3)$ & $17.1526(7)$ & 90. & 90. & 90. & 1.3390 & 1500.1851 \\
$1.03 \mathrm{E}-04^{b}$ & $11.684(6)$ & $7.720(4)$ & $16.659(13)$ & $89.0(1)$ & $90.023(56)$ & $89.915(40)$ & $1.337(1)$ & 1502.423 \\
1 & $11.511(3)$ & $7.155(2)$ & $16.482(6)$ & $88.525(47)$ & $89.894(34)$ & $89.922(18)$ & $1.480(1)$ & 1357.024 \\
2 & $11.385(3)$ & $6.925(2)$ & $16.294(6)$ & $88.364(39)$ & $89.814(25)$ & $89.933(21)$ & $1.564(1)$ & 1284.106 \\
3 & $11.261(4)$ & $6.788(2)$ & $16.126(6)$ & $88.395(31)$ & $89.773(26)$ & $89.938(31)$ & $1.630(1)$ & 1232.172 \\
4 & $11.131(4)$ & $6.705(3)$ & $15.940(7)$ & $88.506(33)$ & $89.758(31)$ & $89.913(32)$ & $1.689(1)$ & 1189.239 \\
\hline
\end{tabular}

${ }^{a}$ Experimental. ${ }^{30}$

${ }^{b}$ No external stress applied.
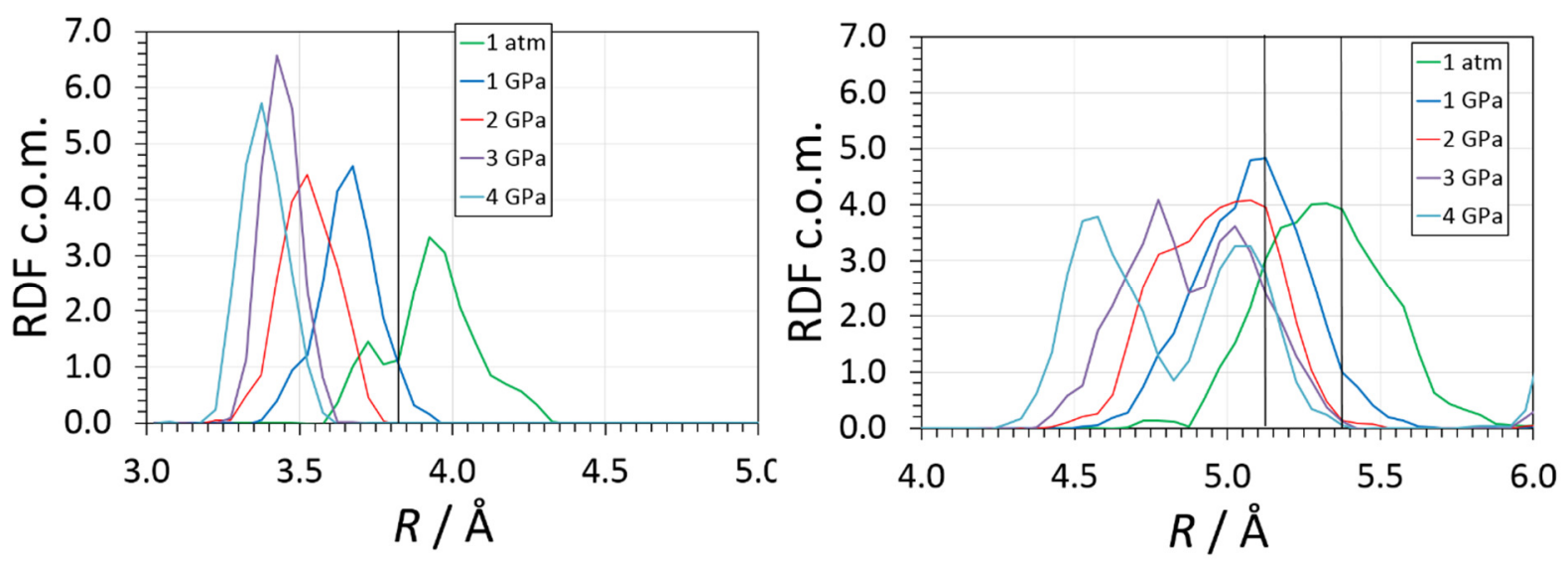

Figure S10. Centre of mass radial distribution functions for the form $\mathrm{I}^{\circ}$ (a) and II (b) of paracetamol, as retrieved from the last frame of $1 \mathrm{~ns}-$ long $N p T$ trajectory under external hydrostatic stress field. Vertical black lines mark the c.o.m. distances in perfect experimental structures at $p=1 \mathrm{~atm}$. 

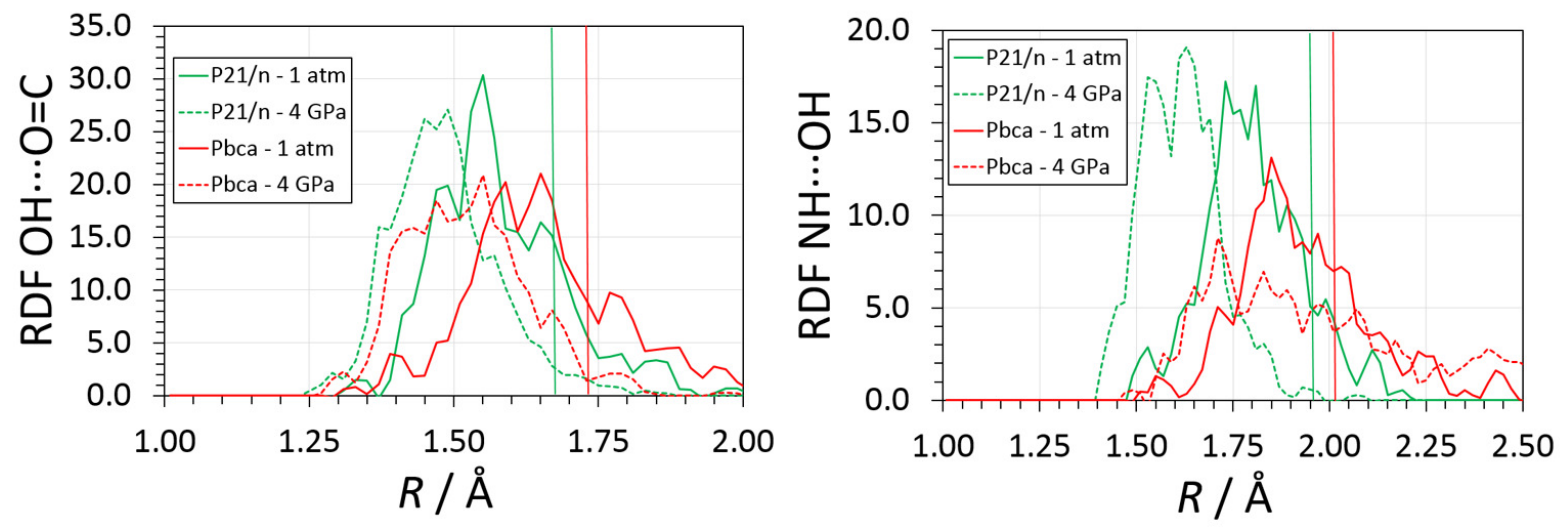

Figure S11. Left: Radial distribution functions (RDF) for the $\mathrm{OH} \cdots \mathrm{O}$ distances, taken from the final frames of the MD trajectories of polymorphs $\mathrm{I}^{\circ}$ (green) and II (red) from 1 atm (full lines) to $4 \mathrm{GPa}$ (dotted lines). Vertical bars correspond to perfect experimental crystal structures. Right: Same, for $\mathrm{NH}^{\cdots} \mathrm{O}$ interactions.

\section{S5. Cohesive energies.}

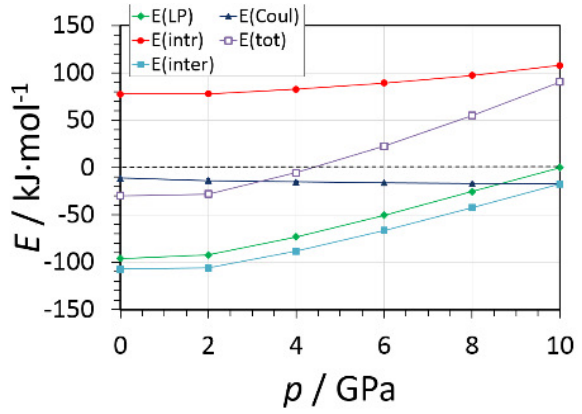

(a)

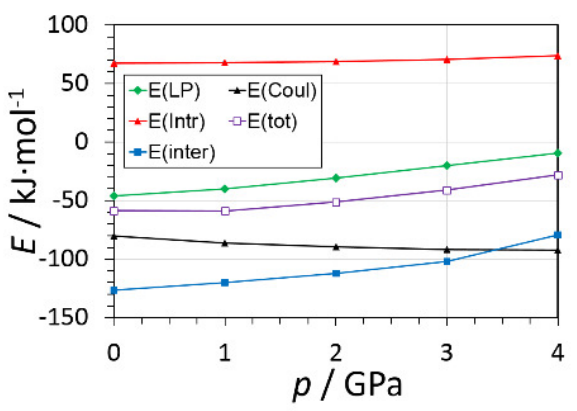

(b)

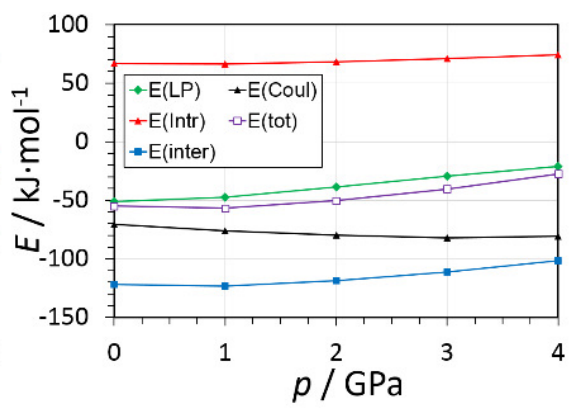

(c)

Figure S12. Decomposition of the total cohesive energy per molecule of anthracene (a) and polymorphs $\mathrm{I}^{\mathrm{O}}$ (b) and II (c) of paracetamol as a function of pressure. All data were obtained by averaging the contributions of the last $200 \mathrm{ps}$ of $1 \mathrm{~ns}-$ long $N p T$ trajectories at $T=300 \mathrm{~K}$ in hydrostatic stress fields. Recall that $E$ (tot) $=E(\mathrm{LP})+E$ (Coul) $+E$ (intr), $E(\mathrm{LP})$ being the dispersive-repulsive potential, $E$ (Coul) the electrostatic one, and $E($ intr) the potential energy contribution due to the intramolecular part of the Force Field. E(inter) highlights the intermolecular part of the potential and is equal to $E(\mathrm{LP})+E(\mathrm{Coul})$.

Comment. As expected, the intermolecular energy of anthracene is dominated by dispersive terms, while in paracetamol the electrostatic interactions clearly provide the most significant contribution to $E$ (inter). Interestingly, Coulomb terms are less dependent on pressure, but show an opposite trend with respect to the $E(\mathrm{LP})$ ones, as they become more attractive at high $p$. This is due to closer contacts of favourable directional interactions, such as $\mathrm{CH} \cdots \pi$ (anthracene) and $\mathrm{H} \cdots \mathrm{O}$ (paracetamol) bonds. 


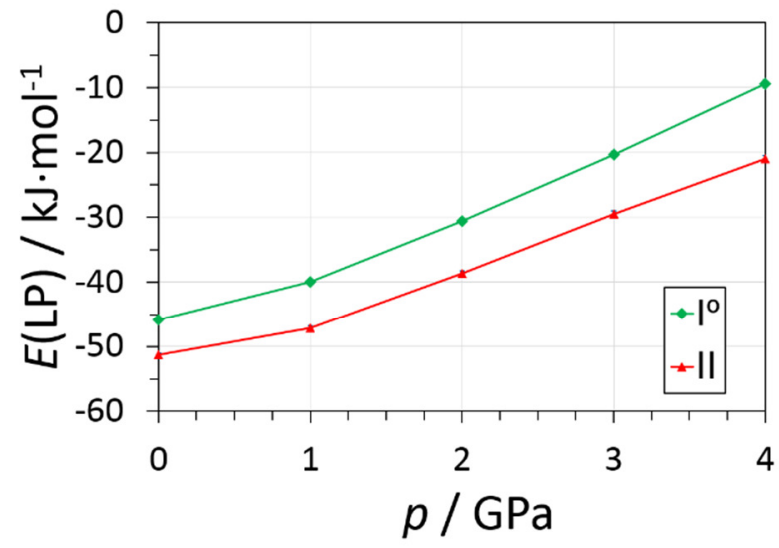

(a)

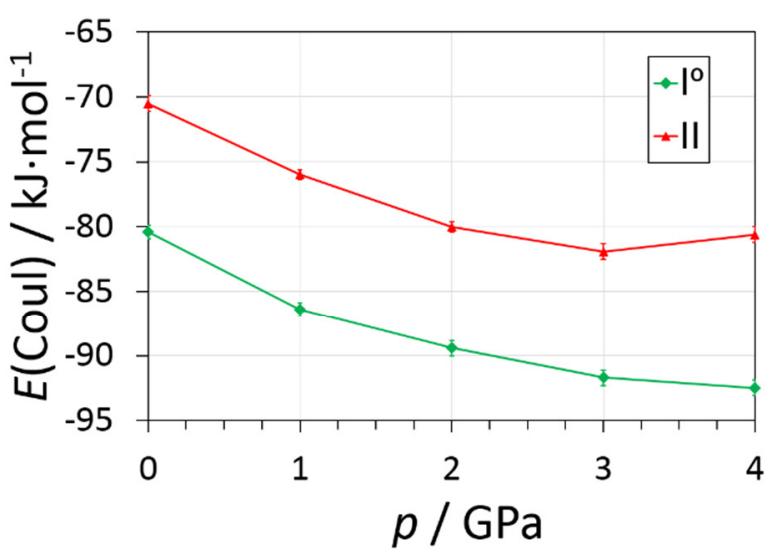

(b)

Figure S13. Direct comparison of dispersive-repulsive (a) and Coulomb (b) contributions to the potential energy of the two polymorphs of paracetamol, I (green rhombi) and II (red triangles) as a function of $\mathrm{p}$. The data points are the same shown in Figure S12 above.

\section{S6. Anisotropic stress field}
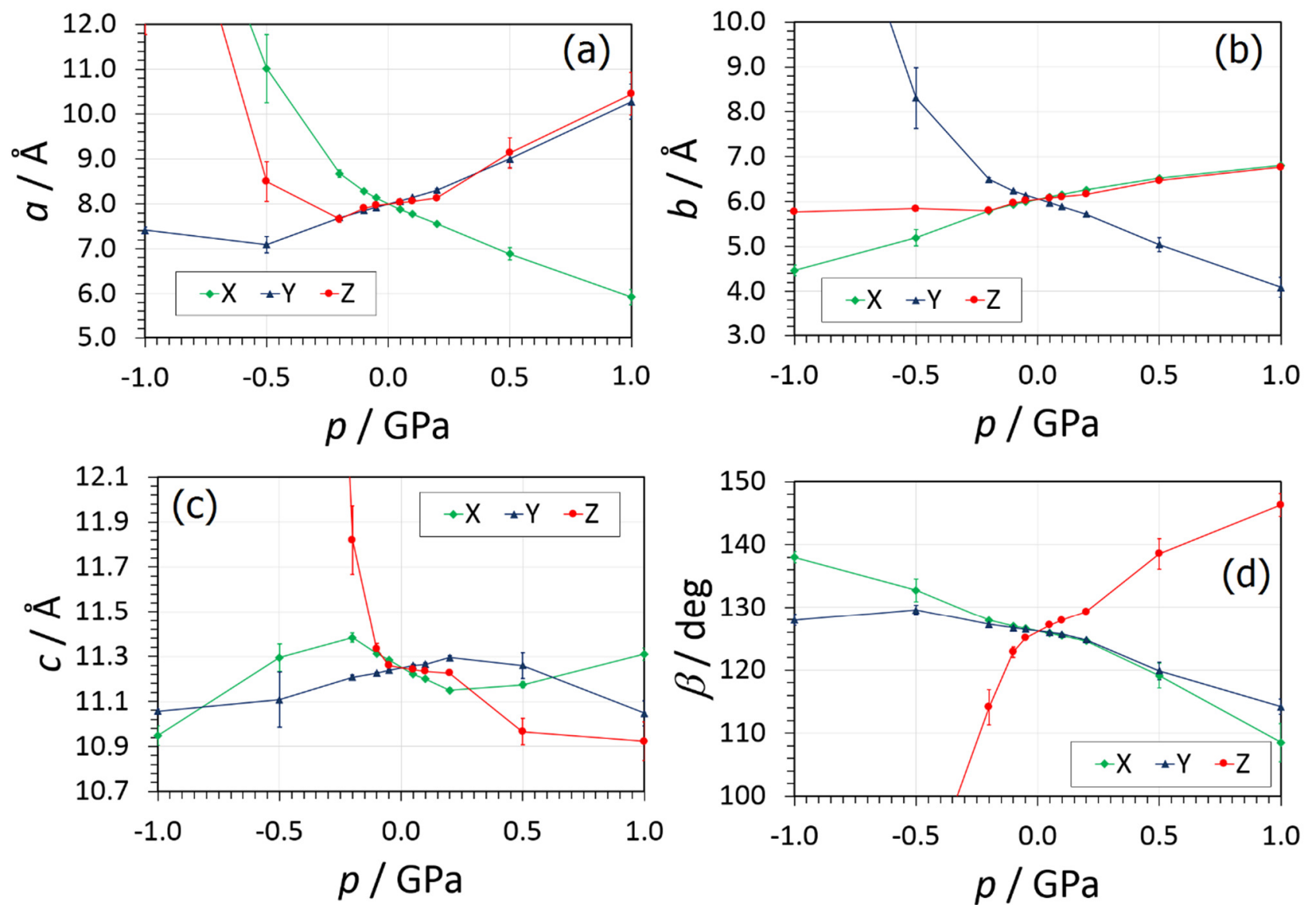

Figure S14. Cell parameters of anthracene averaged over the last $100 \mathrm{ps}$ of $200 \mathrm{ps}-$ long $N p T$ simulations at $T=300 \mathrm{~K}$ under uniaxial stress directed along either the $\mathrm{X}$ (green), Y (blue) or $\mathrm{Z}$ (red) laboratory axis. Broken lines serve only as a guide for the eye. Error bars correspond to 1 estimated standard deviations. Negative pressures mean tensile stress, positive ones compression stress. 

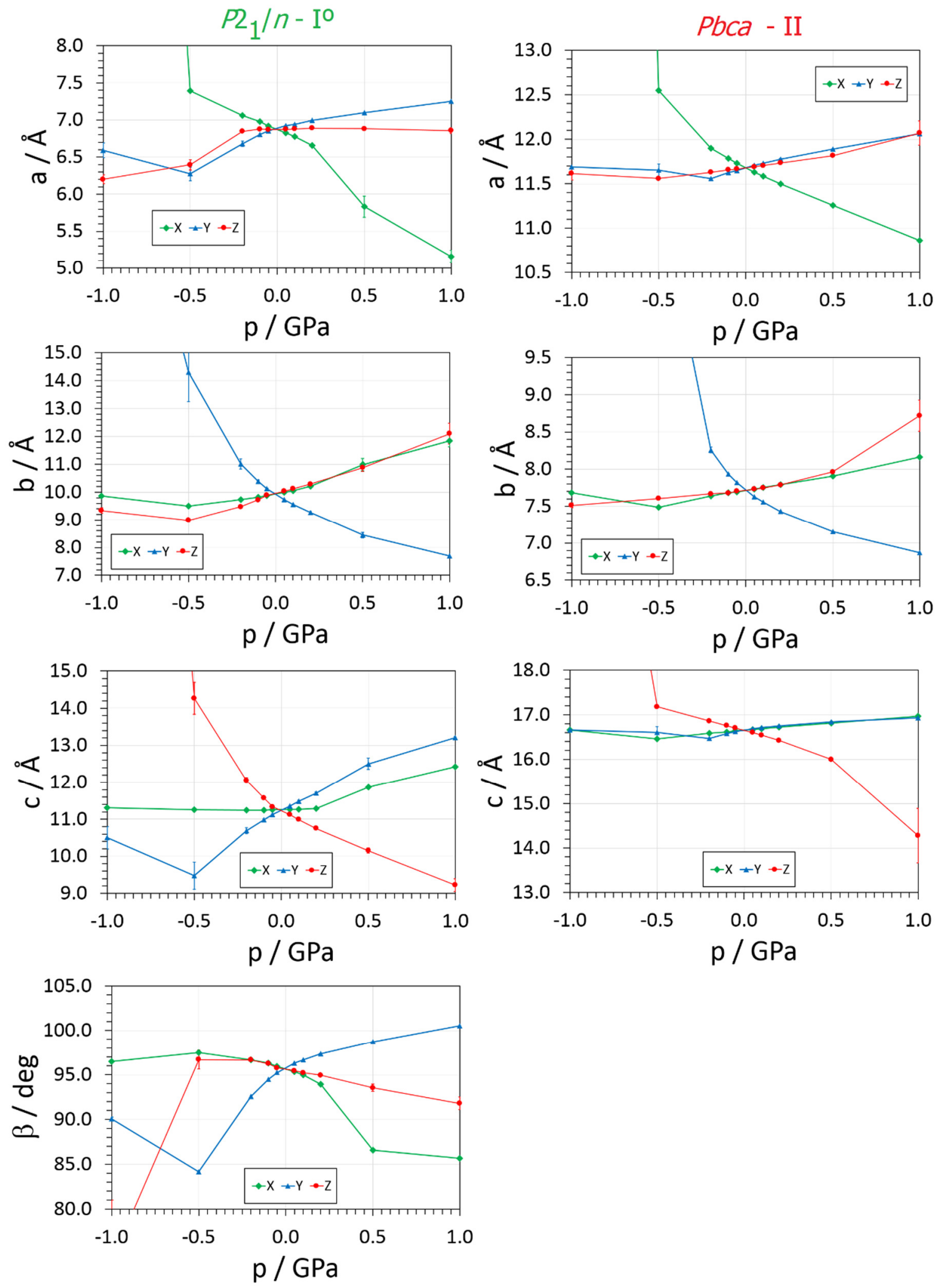

Figure S15. Cell parameters of paracetamol averaged over the last 100 ps of 200 ps-long $N p T$ simulations at $T=300 \mathrm{~K}$ under uniaxial stress directed along either the $\mathrm{X}$ (green), $\mathrm{Y}$ (blue) or Z (red) laboratory axis. Broken lines serve only as a guide for the eye. Error bars correspond to 1 estimated standard deviations. Negative pressures mean tensile stress, positive ones compression stress. Left column: $P 2_{1} / n$ form. Right column: $P b c a$ form. 


\section{REFERENCES}

(1) Parrinello, M.; Rahman, A. Crystal Structure and Pair Potentials: A MolecularDynamics Study. Phys. Rev. Lett. 1980, 45 (14), 1196-1199.

(2) Parrinello, M.; Rahman, A. Polymorphic Transitions in Single Crystals: A New Molecular Dynamics Method. J. Appl. Phys. 1981, 52 (12), 7182-7190.

(3) Braun, E.; Moosavi, S. M.; Smit, B. Anomalous Effects of Velocity Rescaling Algorithms: The Flying Ice Cube Effect Revisited. J. Chem. Theory Comput. 2018.

(4) Gavezzotti, A.; Lo Presti, L. Dynamic Simulation of Liquid Molecular Nanoclusters: Structure, Stability and Quantification of Internal (Pseudo)Symmetries. New J. Chem. 2019, 43 (5), 2077-2084.

(5) Gavezzotti, A.; Lo Presti, L. Molecular Dynamics Simulation of Organic Crystals: Introducing the CLP-Dyncry Environment. J. Appl. Crystallogr. 2019, 52 (6), 12531263.

(6) Besler, B. H.; Merz, K. M.; Kollman, P. A. Atomic Charges Derived from Semiempirical Methods. J. Comput. Chem. 1990, 11 (4), 431-439.

(7) Gavezzotti, A.; Lo Presti, L.; Rizzato, S. Mining the Cambridge Database for Theoretical Chemistry. Mi-LJC: A New Set of Lennard-Jones-Coulomb Atom-Atom Potentials for the Computer Simulation of Organic Condensed Matter. CrystEngComm 2020.

(8) Brütting, W. Physics of Organic Semiconductors; Brütting, W., Ed.; Wiley-VCH Verlag GmbH \& Co. KGaA, 2005.

(9) Horowitz, G. Organic Field-Effect Transistors. Adv. Mater. 1998, 10 (5), 365-377.

(10) Nijegorodov, N.; Ramachandran, V.; Winkoun, D. P. The Dependence of the Absorption and Fluorescence Parameters, the Intersystem Crossing and Internal Conversion Rate Constants on the Number of Rings in Polyacene Molecules. Spectrochim. Acta Part A Mol. Biomol. Spectrosc. 1997, 53 (11), 1813-1824.

(11) Aust, R. B.; Bentley, W. H.; Drickamer, H. G. Behavior of Fused-Ring Aromatic Hydrocarbons at Very High Pressure. J. Chem. Phys. 1964, 41 (6), 1856-1864.

(12) Oehzelt, M.; Resel, R.; Nakayama, A. High-Pressure Structural Properties of Anthracene up to $10 \mathrm{GPa}$. Phys. Rev. B - Condens. Matter Mater. Phys. 2002.

(13) Mason, R. The Crystallography of Anthracene at $95^{\circ} \mathrm{K}$ and $290^{\circ} \mathrm{K}$. Acta Crystallogr. 1964.

(14) Ghanem, C. I.; Pérez, M. J.; Manautou, J. E.; Mottino, A. D. Acetaminophen from Liver to Brain: New Insights into Drug Pharmacological Action and Toxicity. Pharmacological Research. 2016.

(15) Haisa, M.; Kashino, S.; Kawai, R.; Maeda, H. The Monoclinic Form of PHydroxyacetanilide. Acta Crystallogr. Sect. B Struct. Crystallogr. Cryst. Chem. 1976.

(16) Druzhbin, D. A.; Drebushchak, T. N.; Min'kov, V. S.; Boldyreva, E. V. Crystal Structure of Two Paracetamol Polymorphs at $20 \mathrm{~K}$ : A Search for the "StructureProperty" Relationship. J. Struct. Chem. 2015.

(17) Perlovich, G. L.; Volkova, T. V.; Bauer-Brandl, A. Polymorphism of Paracetamol. J. Therm. Anal. Calorim. 2007.

(18) Nicnols, G.; Frampton, C. S. Physicochemical Characterization of the Orthorhombic Polymorph of Paracetamol Crystallized from Solution. J. Pharm. Sci. 1998.

(19) Gaisford, S.; Buanz, A. B. M.; Jethwa, N. Characterisation of Paracetamol Form III with Rapid-Heating DSC. J. Pharm. Biomed. Anal. 2010.

(20) Burger, A. Interpretation of Investigations into Polymorphism | ZUR INTERPRETATION VON POLYMORPHIE UNTERSUCHUNGEN. Acta Pharm. 
Technol. 1982, 28 (1), 1-20.

(21) Perrin, M.-A.; Neumann, M. A.; Elmaleh, H.; Zaske, L. Crystal Structure Determination of the Elusive Paracetamol Form III. Chem. Commun. 2009, No. 22, 3181.

(22) Reiss, C. A.; Mechelen, J. B. van; Goubitz, K.; Peschar, R. Reassessment of Paracetamol Orthorhombic Form III and Determination of a Novel Low-Temperature Monoclinic Form III-m from Powder Diffraction Data. Acta Crystallogr. Sect. C 2018, 74 (3), 392-399.

(23) Shtukenberg, A. G.; Tan, M.; Vogt-Maranto, L.; Chan, E. J.; Xu, W.; Yang, J.; Tuckerman, M. E.; Hu, C. T.; Kahr, B. Melt Crystallization for Paracetamol Polymorphism. Cryst. Growth Des. 2019, 19 (7), 4070-4080.

(24) Smith, S. J.; Bishop, M. M.; Montgomery, J. M.; Hamilton, T. P.; Vohra, Y. K. Polymorphism in Paracetamol: Evidence of Additional Forms IV and V at High Pressure. J. Phys. Chem. A 2014, 118 (31), 6068-6077.

(25) Sibik, J.; Sargent, M. J.; Franklin, M.; Zeitler, J. A. Crystallization and Phase Changes in Paracetamol from the Amorphous Solid to the Liquid Phase. Mol. Pharm. 2014, 11 (4), 1326-1334.

(26) Hendriksen, B. A.; Grant, D. J. W.; Meenan, P.; Green, D. A. Crystallisation of Paracetamol (Acetaminophen) in the Presence of Structurally Related Substances. $J$. Cryst. Growth 1998, 183 (4), 629-640.

(27) Boldyreva, E. V.; Shakhtshneider, T. P.; Vasilchenko, M. A.; Ahsbahs, H.; Uchtmann, H. Anisotropic Crystal Structure Distortion of the Monoclinic Polymorph of Acetaminophen at High Hydrostatic Pressures. Acta Crystallogr. Sect. B Struct. Sci. 2000.

(28) Boldyreva, E. V.; Shakhtshneider, T. P.; Ahsbahs, H.; Sowa, H.; Uchtmann, H. Effect of High Pressure on the Polymorphs of Paracetamol. In Journal of Thermal Analysis and Calorimetry; 2002.

(29) Bouhmaida, N.; Bonhomme, F.; Guillot, B.; Jelsch, C.; Ghermani, N. E. Charge Density and Electrostatic Potential Analyses in Paracetamol. Acta Crystallogr. Sect. B Struct. Sci. 2009, 65 (3), 363-374.

(30) Chan, E. J.; Goossens, D. J. Study of the Single-Crystal X-Ray Diffuse Scattering in Paracetamol Polymorphs. Acta Crystallogr. Sect. B Struct. Sci. 2012, 68 (1), 80-88.

(31) L. u. A. Kofler, Thermo - Mikro - Methoden Zur Kennzeichnung Organischer Stoffe Und Stoffgemische. Verlag Chemie GmbH., Weinheim/ Bergstr. 1954, 608 S., 247 Abb. u. 16 Tafeln, Preis Ganzln. DM 39.80. Fette, Seifen, Anstrichm. 1957, 59 (8), 720-720. 